\title{
Organismos de los arrecifes coralinos de Costa Rica: Descripción, distribución geográfica e historia natural de los corales zooxantelados (Anthozoa: Scleractinia) del Pacífico
}

\author{
Jorge Cortés ${ }^{1}$ y Héctor Guzmán² \\ 1 Museo de Zoología, Escuela de Biología, y Centro de Investigación en Ciencias del Mar y Limnología (CIMAR), Universidad \\ de Costa Rica, 2060 San José, Costa Rica. \\ Smithsonian Tropical Research Institute, Apartado 2072, Balboa, Panamá.
}

Recibido 28-IV-1997. Corregido 06-X-1997. Aceptado 09-X-1997

\begin{abstract}
Twenty two species of zooxanthellate scleractinian corals from the Pacific of Costa Rica are described and illustrated with macrophotographs. Keys to thegeneraand species are included. Their geographic distributions in Costa Rica and world-wide are noted, as well as aspects of the natural history of the species. Sixteen species have wide distributions, from the Red Sea or Indian Ocean to the Pacific coast of America, two species are endemic to the eastern Pacific, four species are found only in the Pacific Ocean; there are no species in common with the Caribbean-Atlantic. Two species, Leptoseris scabra and Pavon xarifea, are new records for the eastern Pacific.
\end{abstract}

Key words: Corals, Eastern Pacific, taxonomy, marine biodiversity.

escleractinios del Pacífico de Costa Rica aparece en el trabajo de Durham y Barnard (1952), donde informan sobre corales encontrados en el Pacífico Oriental durante las expediciones del Velero III y Velero IV de la Fundación Allan Hancock de los Estados Unidos de América. Hertlein (1963), en su publicación sobre biogeografía de la Isla del Coco, menciona los corales registrados por Durham y Barnard (1952) y Durham (1962). Posteriormente, Durham (1966) da una lista más completa y actualizada de las especies de corales de la Isla del Coco en una publicación sobre los corales del Coco y Galápagos, que apareció en un volumen sobre las Islas Galápagos. En 1975, Bakus, publica un trabajo descriptivo de las zonas sublitorales de la Isladel Coco en el que menciona algunasespecies de corales. Para esa mismaépoca y posteriormente (1975 y 1978), Glynn y colaboradores trabajaron en los arrecifes del Pacífico de Costa Rica (Glynn et al. 1983). Ellos estudiaron la muerte de corales hace unos 400 años durante la Pequeña Edad de Hielo, evidente todavíaen arrecifes muertosen Guanacaste. Existe además, una lista inédita de corales para el Parque Nacional deManuel Antonio(Dubois\& Hatziolos 1982). En 1985, Cortés y Murillo publican un trabajo sobre las comunidades coralinas y arrecifes del Pacífico de Costa Rica y dan una lista de especies de corales escleractinios encontrados. Guzmán (1986) incluye una lista de corales de los arrecifes de la Isla del Caño. Guzmán y Cortés (1989a) describen los arrecifes y la distribución de corales de la Isla del Caño, y en una publicación posterior (Guzmán \& Cortés 1989b) presentan las tasas de crecimiento de ocho de esas especies. Hoeksema (1989) en su trabajo sobre los corales hongos (Familia Fungiidae) indica la presencia de dos especiesen Costa Rica. En la década de los 1990s se publican listas de corales de Golfo Dulce (Cortés 1990), Isla del Coco (Guzmán \& Cortés 1992), Parque Nacional Corcovado, Península de Osa (Cortés \& Jiménez 1996), y del 
Area de Conservación Guanacaste (Cortés 19961997a). Cortés (1996-1997b) presenta una recopilación de todos los Cnidarios informados para Costa Rica, donde se incluyen todos los corales.

Los corales del Pacífico Oriental son muy diferentes a los del Caribe, y están emparentados con los corales de la Provincia biogeográfica del Indo-Pacífico(Cortés 1986).En total se reconocen 33 especies, en 11 géneros, de corales formadores de arrecifes e hidrocorales (Glynn 1997). Para el Pacífico de Costa Rica se han identificado 22 especies de corales formadores de arrecifes, 25 especies de corales ahermatípicos, y ninguna especie de hidrocoral (Cortés 1996-1997b).

El presente trabajo tiene como objetivo describir las veinte y dos especies de corales escleractinios zooxantelados encontrados hasta el momento en el Pacífico de Costa Rica (Cuadro 1), para lo cual se hará referencia a las siguientes publicaciones: Durham y Barnard (1952), Wells (1956, 1982, 1983), Veron y Pichon (1976, 1979, 1982), Veron et al. (1977), Maragos (1977), Dinesen (1980), Scheer y Pillai (1983), Scott (1984), Veron y Wallace (1984), Prahl y Erhardt (1985), Nemenzo (1986), Veron (1986, 1992, 1993), Veron y Kelley (1988), Hoeksema (1989), Sheppard y Sheppard (1991). Se incluye además, información sobre la distribución geográfica de cada especie a nivel mundial, basada en las referencias anteriores, y para Costa Rica, basado en Cortés y Murillo (1985), Guzmán y Cortés (1989a, 1992), Cortés (1990, 1996-1997a), y Cortés y Jiménez (1996). Las localidades mencionadas en Costa Rica se indican en la Fig. 1. Finalmente, se describen algunos aspectos sobre la historia natural de las especies.

\section{CUADRO}

Lista de especies de corales escleractinios zooxantelados encontrados en el Pacífico de Costa Rica

SUBCLASE Zoantharia de Blainville, 1830 ORDEN Scleractinia Bourne, 1900

SUBORDEN Astrocoeniina Vaughan \& Wells, 1943

FAMILIA POCILLOPORIDAE Gray, 1842

1. Pocillopora capitata Verrill, 1864

2. Pocillopora damicornis (Linnaeus, 1758)

3. Pocillopora elegans Dana, 1846

4. Pocillopora eydouxi Milne Edwards \& Haime, 1860

5. Pocillopora meandrina Dana, 1846

SUBORDEN Fungiina Verrill, 1865

FAMILIA PORITIDAE Gray, 1842

6. Porites lobata Dana, 1840

7. Porites panamensis Verrill, 1866

8. Porites (Synarea) rus (Forskål, 1775)

FAMILIASIDERASTREIDAE Vaughan \& Wells, 1943

9. Psammocora obtusangula (Lamarck, 1816)

10. Psammocora stellata (Verrill, 1866)

11. Psammocora superficialis (Gardiner, 1898)

FAMILIA AGARICIIDAE Gray, 1847

12. Gardineroseris planulata (Dana, 1846)

13. Leptoseris papyracea (Dana, 1846)

14. Leptoseris scabra Vaughan, 1907

15. Pavona clavus (Dana, 1846)

16. Pavona frondifera (Lamarck, 1816)

17. Pavona gigantea Verrill, 1869

18. Pavona maldivensis (Gardiner, 1905)

19. Pavona varians Verrill, 1864

20. Pavona xarifae Scheer \& Pillai, 1974

FAMILIA FUNGIIDAE Dana, 1846

21. Fungia (Cycloseris) curvata (Verrill, 1870)

22. Fungia (Cycloseris) distorta Michelin, 1842

\section{CLAVE PARA LOS GENEROS Y ESPECIES}

La primera parte de esta sección consiste de una clave para los géneros con varias especies y para una especie de género monoespecífico. La segunda parte es una serie de claves para las especies de géneros multiespecíficos. 
Se incluyen estas claves con la idea de actualizar y facilitar el estudio de la biodiversidad de escleractinios. Las claves son basadas principalmente en el análisis de los múltiples especímenes por especiedepositados en el Museo de Zoología de la Universidad de Costa Rica. Es posible que con las nuevas técnicas de análisis, en particular las técnicas moleculares, se describan o redescriban nuevas especies para la región.

1. Pólipos individuales Fungia

Colonias de pólipos . .2

2. Colonias ramificadas

Colonias masivas o incrustantes 5

3. Ramas cortas y gruesas o en forma de nódulos Psammocora

Ramas cortas y delgadas o largas y gruesas .4

4. Cálices solamente de un lado Leptoseris

Cálices todo alrededor de las ramas Pocillopora

5. Cálices de menos de $2 \mathrm{~mm}$ de diámetro y poco profundos ... Porites

Cálices de más de $2 \mathrm{~mm}$ de diámetro y profundos ....... 6

6. Cálices con pocos septos y gruesos Pavona

Cálices con muchos septos y delgados Gardineroseris planulata

\section{CLAVE PARA LAS ESPECIES DE Pocillopora}

I. Ramas de menos de $10 \mathrm{~cm}$ de largo y delgadas.......2 Ramas de más de $10 \mathrm{~cm}$ de largo y gruesas 3

2. Verrugas en las ramas Pocillopora capitata

Ramas sin verrugas prominentes Pocillopora damicornis
Ramas con terminaciones aplanadas

Pocillopora meandrina

4. Gran cantidad de verrugas a lo largo de las ramas, que pueden al canzar largos de $20 \mathrm{~cm}$ y diámetros de 2 a $3 \mathrm{~cm}$ Pocillopora elegans

Verrugas presentes pero en bajas densidades; las ramas pueden alcanzar largos de hasta $40 \mathrm{~cm}$ y diámetros de 4 a $6 \mathrm{~cm}$. Pocillopora eydouxi

\section{CLAVE PARA LAS ESPECIES DE Porites}

1. Coralitos muy pequeños, menos de $1 \mathrm{~mm}$.....Porites rus Coralitos de más de $1 \mathrm{~mm}$ 2

2. Separaciones entre los cálices elevados, coralitos hasta de $1 \mathrm{~mm}$............................................ orites panamensis

Separaciones entre los cálices poco pronunciadas, coralitos de 1-2 mm, colonias grandes. Porites lobata

\section{CLAVE PARA LAS ESPECIES DE Psammocora}

1. Ramas muy delgadas, menos de $2 \mathrm{~mm}$ Psammocora obtusangula

Ramas de más de 2 mm

2. Coralitos bien definidos, ramas gruesas o colonias incrustantes Psammocora superficialis

Coralitos difusos, ramas de 5 a $10 \mathrm{~mm}$ de grueso Psammocora stellata

\section{CLAVE PARA LAS ESPECIES DE Leptoseris}

Colonias de ramas delgadas, despegada de sustrato ....... Leptoseris papyracea

Colonias gruesas e incrustantes .......... Leptoseris scabra 


\section{CLAVE PARA LAS ESPECIES DE Pavona}

1. Colonias hemisféricas y laminares 2

Colonias incrustantes Pavona varians

2. Cálices gdes. (más de $1.5 \mathrm{~mm}$ de diámetro 3

Cálices pequeños (menos de $1.5 \mathrm{~mm}$ Pavona maldivensis

Cálices no levantados Pavona gigantea

4. Forma de la colonia lobulada Pavona clavus

Colonias no lobuladas 5

5. Colonias con estructuras esqueléticas levantadas Pavona frondifera

Superficie de la colonia relativamente lisa

Pavona xarifae

\section{CLAVE PARA LAS ESPECIES DE Fungia}

Esqueleto relativamente grueso, denso, y curvado Fungia (Cycloseris) curvata

Esqueleto generalmente con fisuras, frágil, y relativamente plano Fungia (Cycloseris) distorta

\section{DESCRIPCION DE LAS ESPECIES, DISTRIBUCION E HISTORIA NATURAL}

\section{Pocillopora capitata Verrill, 1864}

\section{DESCRIPCION:}

Coral de ramificaciones delgadas (menos de 2 $\mathrm{cm})$ y cortas (menos de $10 \mathrm{~cm}$ ), con verrugas puntiagudas. Los cálices son muy sencillos ya que no hay septos u otras estructuras. Especie poco abundante, crece principalmente en plataformas, zonas de alta energía y lagunas someras. Fig. 2 a, b.

\section{DISTRIBUCIONGEOGRAFICA:}

Costa Rica: Bahía Culebra, Sámara, Manuel Antonio, Isla del Caño.

Resto del mundo: Endémica del Pacífico Oriental (Wells 1983).

\section{Pocillopora damicornis (Linnaeus, 1758)}

\section{DESCRIPCION:}

Coral de ramificaciones delgadas (menos de 2 $\mathrm{cm})$ y generalmente cortas (menos de $15 \mathrm{~cm}$ ), sin verrugas. Los cálices son de 0,5 a $1,5 \mathrm{~mm}$ de diámetro y generalmente no tienen estructuras internas, e.g., septos o columnela, o están muy reducidas. Generalmente el tejido es amarillo. Una de las especie más abundantes. Se puede encontraren forma aislada o formando estructuras arrecifales compactas. La variabilidad de la forma de la colonia es alta. Fig. 3 a, b.

\section{DISTRIBUCIONGEOGRAFICA:}

Costa Rica: Archipiélago de las Islas Murciélago, Bahía Culebra, Bahía Brasilito, Sámara, Cabo Blanco, Punta Leona, Herradura, Manuel Antonio, Punta Uvita, Península de Osa, Golfo Dulce, Isla del Caño, Isla del Coco.

Resto del mundo: especie de distribución muy amplia, Mar Rojo, Océano Indico y el Océano Pacífico (Veron 1993).

\section{Pocillopora elegans Dana, 1846}

\section{DESCRIPCION:}

Coral de ramificaciones de grosor mediano (entre 2 y $4 \mathrm{~cm}$ ) y largas (más de $15 \mathrm{~cm}$ ), con verrugas a todo lo largo de las ramas. Los cálices tienen septospequeños y la columnelaestá ausente o es pequeña. Tejido generalmente amarillo pero 
puedeserverde también. Especie muy abundante. Fig. 4 a, b.

\section{DISTRIBUCIONGEOGRAFICA:}

Costa Rica: Archipiélago de las Islas Murciélago, Bahía Culebra, Península de Osa, Isla del Caño, Isla del Coco.

Resto del mundo: Filipinas (Nemenzo 1986; Veron \&Hodgson 1989), Guam(Randall \& Myers 1983), Enewetak (Devaney \& Lang 1987), Polinesia Francesa (Pichon 1985), Pacífico Oriental (Wells 1983).

\section{Pocilloporaeydouxi MilneEdwards \& Haime, 1860}

\section{DESCRIPCION:}

Coral de ramificaciones gruesas (más de $4 \mathrm{~cm}$ ) y largas (más de $20 \mathrm{~cm}$ ), con verrugas a todo lo largo de las ramas. Las estructuras dentro de los cálices varía en una misma rama, hay septos y columnela. Especie poco abundante. Fig. 5 a, b.

\section{DISTRIBUCION GEOGRAFICA:}

Costa Rica: Península de Santa Elena, Archipiélago de las Islas Murciélago, Bahía Culebra, Península de Osa, Isla del Caño, Isla del Coco.

Resto del mundo: Especie de distribución amplia, del Mar Rojo y la costa este de Africa hasta las costas de América Central (Veron 1993).

\section{Pocillopora meandrina Dana, 1846}

\section{DESCRIPCION:}

Coral de ramificaciones de grosor (entre 2 y 4 $\mathrm{cm}$ ) y largo mediano (entre 10 y $15 \mathrm{~cm}$ ), con verrugas a todo lo largo de las ramas. El extremo de las ramas es característicamente aplanado; la parte superior tiene todos los cálices unidos (llamados cálices celulares en Veron \& Pichon 1976). Especie poco abundante. Fig. 6 a, b.

\section{DISTRIBUCIONGEOGRAFICA:}

Costa Rica: Península de Santa Elena, Archipiélago de las Islas Murciélago, Bahía Culebra, Isla del Coco.

Restodel mundo: Desde las Islas Nicobar en el Océano Indico hasta la costa Pacífica de América Central (Veron 1993).

\section{HISTORIA NATURAL DE LAS ESPECIES DEL GENERO Pocillopora:}

Todas las especies de Pocillopora son llamadas en Costa Rica por los pobladores de la costa, cirios, y los arrecifes formados por este género ciriales. Este nombre se debe a lo parecido en la forma de estas especies con los cirios de las iglesias.

Es el género con la segunda mayor diversidad de especies y considerando uno de los principales constructores de arrecifes en la región del Pacífico Oriental (Glynn \& Wellington 1983; Guzmán \& Cortés 1993). Pocillopora se ha encontrado, en el Pacifico Oriental, a más profundidad que otras especies de corales zooxantelados (Guzmán 1986). En Costa Rica, estas especies formaron grandes arrecifes a lo largo de toda la costa, principalmente en zonas pocas profundas donde formaron bajos (Glynn et al. 1983; Guzmán \& Cortés 1989a; Cortés 1996-1997a). Esos arrecifes murieron durante la Pequeña Edad de Hielo hace unos 400 años (Glynn et al. 1983). Actualmente, la mayor parte de los arrecifes de Pocillopora están muertos y cubiertos por algas coralinas incrustantes (Glynn et al. 1983; Cortés 19961997a). En el pasado, algunasespecies de singular belleza como $P$. eydouxi y $P$. meandrina, abundaban en la región norte del país, pero sus poblaciones fueron reducidas dramáticamente debido a la extracción de colonias para venderlas a los turistas (Cortés \& Murillo 1985). 
Estas especies poseen la mayor tasa $\mathrm{de}$ crecimiento en los corales de la región (hasta 3.5 cm/año en Costa Rica, Guzmán \& Cortés 1989b) y compiten agresivamente por espacio con el resto de las especies, principalmente con las de crecimiento lento. El principal mecanismo de reproducción es asexual o por fragmentación (Glynn et al. 1991). La reproducción sexual varía con la especie, y ocurre más frecuentemente durante las lunas nueva y llena. Pocillopora damicornis, la más importante y abundante de todas las especies, se reproduce, tanto en la época secacomo en la lluviosa, mientras que $P$. elegans se reproduce principalmente a mediados de la época Iluviosa. Los corales Pocillopora del PacíficoOriental son de fertilización externa y no liberanlarvas plánulascomolohacen las especies en la mayor parte de los arrecifes del Pacífico (Glynn et al. 1991). A pesar de que se han observado gametos de ambos sexos durante todo el año, la tasa de reclutamiento por medio de larvas es casi nula para los arrecifes de la región (Richmond 1985). Los espermatozoides de Pocillopora damicornis y de $P$. elegans tienen núcleos en forma de bala y mitocondrias elongadas; característica distintiva del suborden Astrocoeniina (Steiner \& Cortés 1996).

Pocillopora spp. son depredadas por diversos organismos entre los cuales destacan principalmente el pez timboril Arothron meleagris, los gastrópodos Quoyula monodonta (Coralliophila madrepora) y Jenneria pustulata, los cangrejos hermitaños Aniculus elegans y Trizopagurus magnificus, y la estrella de mar Acanthaster planci (Glynn \& Wellington 1983, Guzmán 1988, Guzmán \& Robertson 1989).Esta última, aunque poco abundante en Costa Rica, se ha observado depredando colonias aisladas de Pocillopora. Estas colonias no tenían loscangrejos Trapezia spp. ni el camarón Alpheus lottini, guardianes simbiontes de Pocillopora, que defienden los corales de Acanthaster, la estrella comedora de coral (Glynn 1983).

El género Pocillopora haestado presente desde el inicio de la formación de los arrecifes recientes hace unos 5500 años (Cortés et al. 1994). Los espesores de esos arrecifes oscila entre 0.6 y 13.4 m en la costa de Costa Rica e islas oceánicas (Macintyre et al. 1992; Cortés et al. 1994).

\section{Porites lobata Dana, 1846}

\section{DESCRIPCION:}

Coral masivo de cálices pequeños separados por esqueleto reticulado. Las colonias pueden alcanzargran tamaño(varios metros de diámetro), y generalmente son pardas. Especie muy abundante en la parte sur de la costa de Costa Rica. Fig. 7 a, b.

\section{DISTRIBUCION GEOGRAFICA:}

Costa Rica: Bahía Culebra, Bahía Brasilito, Sámara, Cabo Blanco, Punta Leona, Manuel Antonio, Punta Uvita, Península de Osa, Golfo Dulce, Isla del Caño, Isla del Coco.

Resto del mundo: especie de distribución muy amplia, Mar Rojo (Sheppard \& Sheppard 1991), y desde Mozanbique hasta las costas de América (Veron 1993).

\section{Porites panamensis Verrill, 1866}

\section{DESCRIPCION}

Colonias pequeñas, verde brillante en la parte norte de la costa, donde es abundante, y gris en la parte sur de Costa Rica. Cálices pequeños $(<0,5$ mm). Fig. 8 .

\section{DISTRIBUCIONGEOGRAFICA:}

Costa Rica: Area de Conservación Guanacaste, Bahía Culebra, Sámara, Cabo Blanco, Isla del Caño.

Oceano Pacífico: Endémica del Pacífico Oriental (Wells 1983). 


\section{Porites (Synarea) rus (Forskål, 1775)}

\section{DEŚCRIPCION:}

Colonias pequeñas, con cálices muy pequeños $(<0.7 \mathrm{~mm})$, casi indistinguibles. Especie muy poco abundante. Fig. 9.

\section{DISTRIBUCIONGEOGRAFICA:}

\section{Costa Rica: Sámara.}

Resto del mundo: Desde el Mar Rojo hasta Costa Rica (Veron 1993).

\section{COMENTARIOS:}

Esta especie, en el Pacífico Oriental, se había encontrado únicamente en Sámara en 1983. No se ha vuelto a observar después de El Niño $1982-$ 1983.

\section{HISTORIA NATURAL DE LAS ESPECIES DEL GENERO Porites:}

Porites lobata en Costa Rica es llamada vernacularmente núcura; se desconoce la etimología de esta palabra.

De todas las especies, Porites lobata podría considerarse como la principal formadora de arrecifes para Costa Rica. La especie domina en más de un $90 \%$ en los arrecifes mejordesarrollados del país: Isladel Caño (Guzmán \& Cortés 1989a), Punta Islotes, Golfo Dulce (Cortés 1990), Isla del Coco (Guzmán \& Cortés 1992). Actualmente los arrecifes de la Isla del Caño son los únicos que presentan una cobertura relativamente alta de coral vivo, ya que los de la Isla del Coco y Punta Islotes en Golfo Dulce se encuentran muy deteriorados por causas naturales o antropogénicas, respectivamente.

La edad de algunos de los arrecifes formados por Porites lobata oscila entre los 5500 y 500 años (Cortés et al. 1994). Estaespecie es formadora de arrecifes o comunidades coralinas en Isla Malpelo, Colombia (Birkeland et al. 1975), Islas
Galápagos (Glynn \& Wellington 1983), e Isla Clipperton (Glynn et al. 1996).

Porites lobata es de crecimiento lento $(1 \mathrm{~cm} /$ año para Costa Rica) aunque muy rápido durante los primeros años (1.4 - 1.9 cm/año: Guzmán \& Cortés 1989b). En algunos arrecifes las colonias puedenalcanzarhasta $3 \mathrm{~m}$ de alto.Lareproducción sexual de $P$. lobata se da a mediados de la época lluviosa, cuando los gametos son liberados en el agua. En la Isla del Caño se encontraron colonias hermafroditas (Glynn et al. 1994). Se piensa que en ciertos arrecifes de la región $P$. lobata domina debido a que es fragmentada continuamente por el pez balístido Pseudobalistes naufragium; algunos de esos fragmentos sobreviven (Guzmán \& Cortés 1989a). Porites panamensis, a pesar de producir larvas (Smith 1991), noes muy abundante en Costa Rica. Esta especie es más abundante en la parte norte del país que en la parte sur (Cortés 1996-1997a). Se conocen muy pocos depredadores de Porites para Costa Rica. Se ha observado a Acanthaster planci depredando a esta especie únicamente en la Isla del Coco, posiblemente debido a la ausencia de sus presas preferidas (Guzmán \& Cortés 1992). La alta densidad de bivalvos Lithophaga spp. que se observa en Porites lobata, estimula a los peces balístidos que se alimentan de estos, los cuales causan heridas en las colonias. Dichas heridas regeneran relativamente rápido sin deteriorar las colonias, y por lo contrario, pareciera que este proceso ayuda en la formación de múltiples fragmentos o clones que al cabo de cierto tiempo se transforman en nuevas colonias si sobreviven. P. lobata es parte de la dieta del pez Arothoron meleagris (Guzmán \& Robertson, 1989), pero su efecto en las colonias no es considerable, como lo es el de $A$. planci, la cual es capaz de destruir colonias enteras.

Dos morfos de Poriteslobatase han observado: uno es de superficie de la colonia lisa y el otro morfo es de superficie con lóbulos de 1 a $10 \mathrm{~cm}$ de diámetro en la base y hasta $10 \mathrm{~cm}$ de alto. Sin embargo, Weil (1992) no los separó, pero si suguiere que las poblaciones de Porites 
panamensis analizadas podrían representar dos especies.

\section{Psammocora obtusangula (Lamarck, 1816)}

\section{DESCRIPCION:}

Colonia de nódulos muy delgados. De 6 a 12 septos denticulados según si están fusionados o no. Columnela prominente a la que llegan los septos. Colonias vivas son pardo claro. Forma parches monoespecíficos de varios metros cuadrados en Golfo Dulce. Fig. 10.

\section{DISTRIBUCION GEOGRAFICA:}

Costa Rica: Golfo Dulce, Isla del Caño.

Resto del Mundo:

\section{Psammocora stellata (Verrill, 1866)}

\section{DESCRIPCION:}

Colonia de nódulos de 1 a $2 \mathrm{~cm}$ de diámetro. Cálices poco diferenciados, con 20 septos denticulados. Generalmente son pardo claro. Especie muy abundante. Fig. 11 a, b.

\section{DISTRIBUCION GEOGRAFICA}

Costa Rica: Archipiélago de las Islas Murciélago, BahíaCulebra, BahíaBrasilito, Cabo Blanco, Punta Mala, Manuel Antonio, Península de Osa, Golfo Dulce, Isla del Caño, Isla del Coco.

Resto del mundo: Guam ((Randall \& Myers 1983), Hawaii (Maragos 1977), Pacífico Oriental (Wells 1983).
11. Psammocora superficialis Gardiner, 1898

\section{DESCRIPCION:}

Colonias con nódulos de más de $2 \mathrm{~cm}$ de diámetro o incrustantes. Coralitos distribuidos en forma más o menos regular sobre el esqueleto. Los septos están fusionados a la columnela. Los cálices se distinguen mejor que en las otras especies de este género. Fig. 12 a, b.

\section{DISTRIBUCION GEOGRAFICA:}

Costa Rica: Península de Osa, Isla del Caño, Isla del Coco.

Resto del mundo: Distribución muy amplia, el Mar Rojo (Sheppard \& Sheppard 1991), el Golfo Pérsico (Hodgson \& Carpenter 1995) y desde la costa Africanahastael Pacífico de América(Veron 1993).

\section{HISTORIA NATURAL DE LAS ESPECIES DEL GENERO Psammocora:}

Especies oportunistas abundantes en ciertos arrecifes de Costa Rica. Se distribuye en casi todas las zonas del arrecife, pero preferiblemente en ambientes de baja energía. Su crecimiento es muy lento $(0.6 \mathrm{~cm} / a n ̃ o$ para Costa Rica: Guzmán \& Cortés 1989b), sin embargo, se reproduce muy rápidamente. La reproducción sexual es importante, pero la reproducción asexual o por fragmentación es más efectiva para dispersarse por el arrecife y colonizar áreas desnudas. Psammocora es depredada por Acanthasterplanci y el pez Arothoron meleagris (Guzmán 1988, Guzmán \& López 1991). Este último tiene una clara preferencia por el coral (Guzmán \& Robertson 1989; Guzmán \& López 1991), y podría reducir las poblaciones del coral en ausencia de otras especies como Porites lobata. 


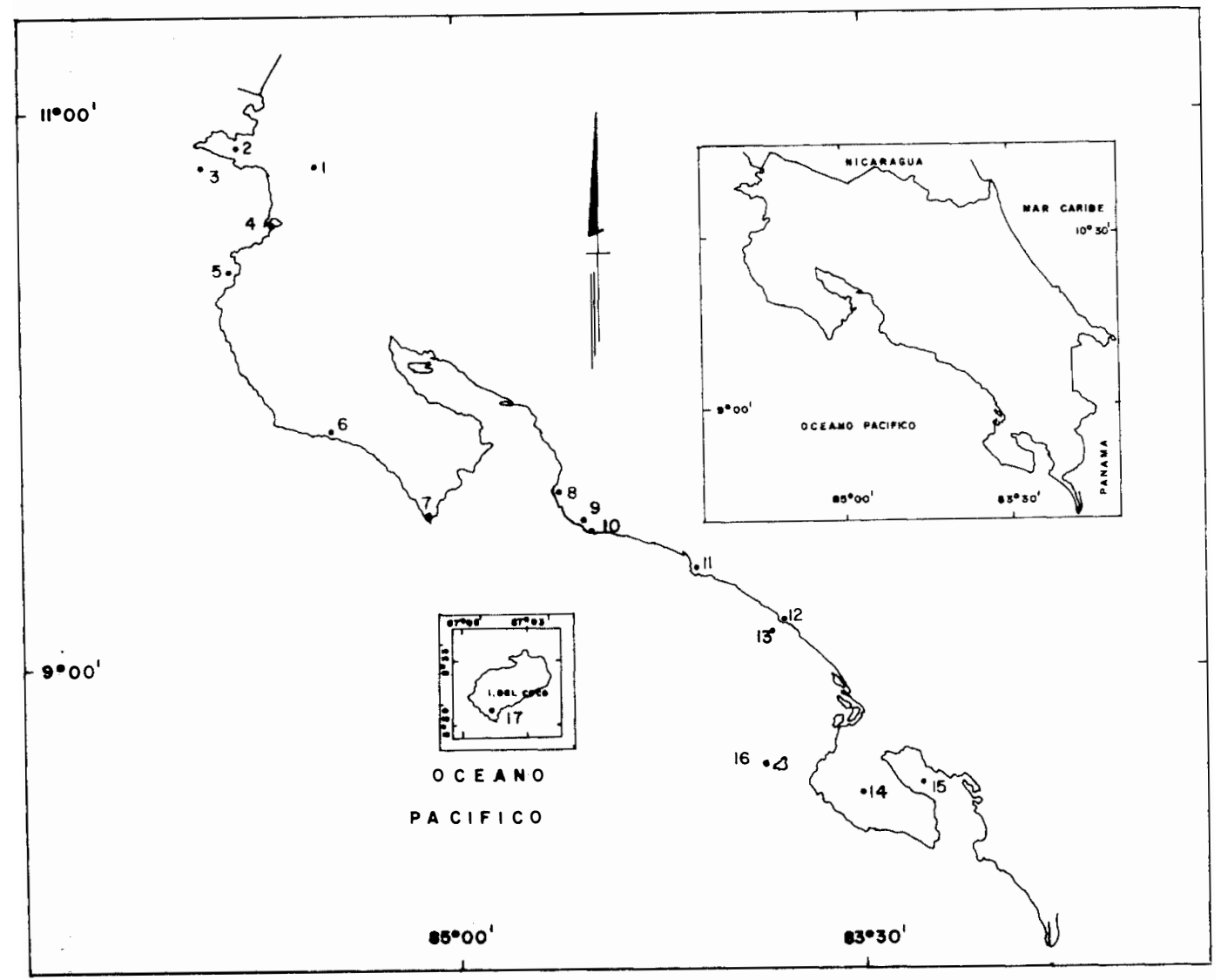

Fig. 1. Costa Pacífica de Costa Rica, con indicación de las localidades mencionadas en el texto.: 1) Area de Conservación Guanacaste, 2) Península de Santa Elena, 3) Archipiélago de las Islas Murciélago, 4) Bahía Culebra, 5) Bahía Brasilito, 6) Sámara, 7) Reserva Absoluta Cabo Blanco, 8) Punta Leona, 9) Herradura, 10) Punta Mala, 11)ParqueNacional Manuel Antonio, 12) Punta Uvita, 13) Parque Marino Ballena, 14) Península de Osa, 15) Golfo Dulce, 16) Isla del Caño, 17) Isla del Coco. 

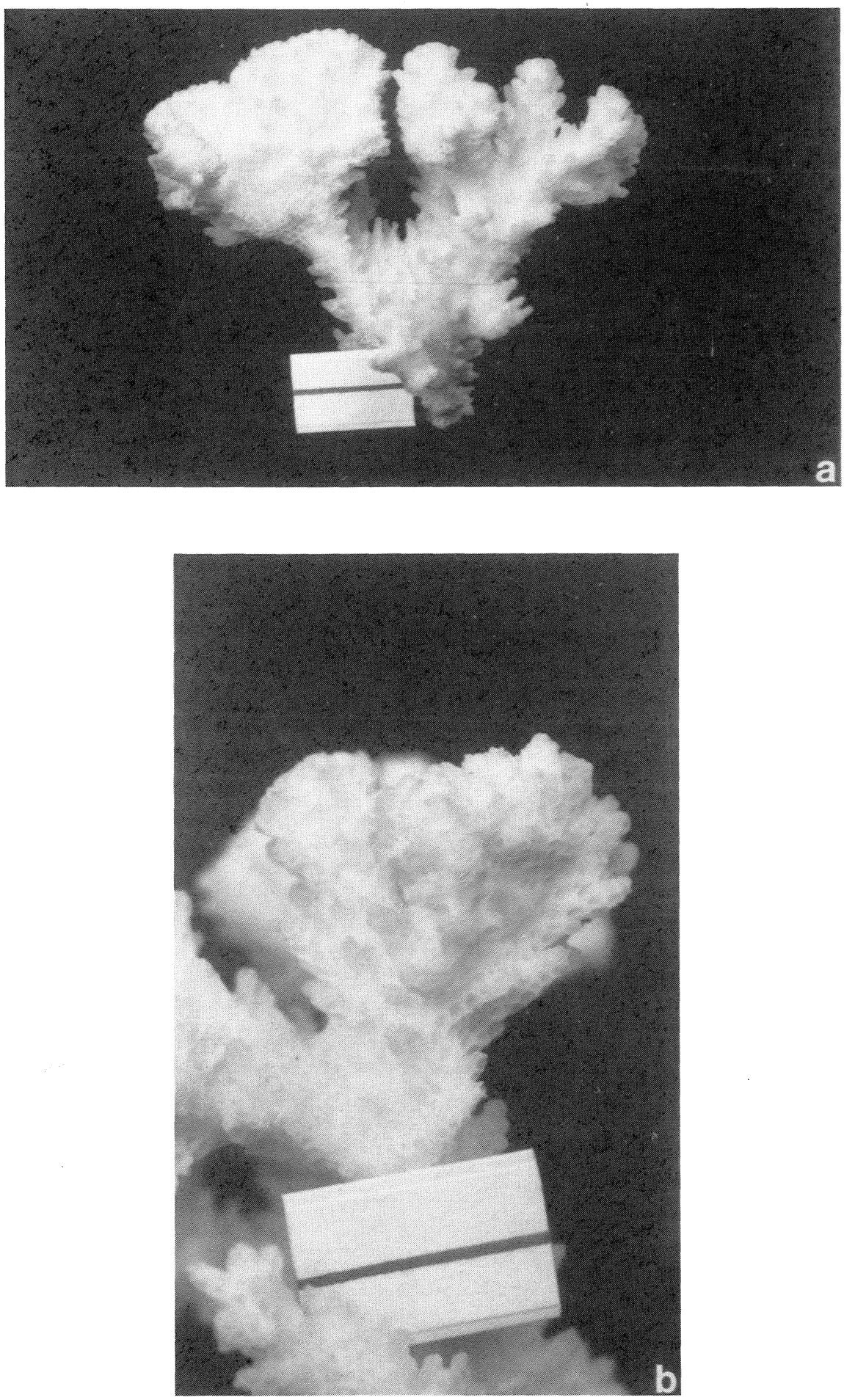

Fig. 2. a) Pocillopora capitata (UCR 700), Sámara, Guanacaste, profundidad $6 \mathrm{~m}, 25 . \mathrm{IV} .1992$, escala $=2 \mathrm{~cm}$; b) detalle, escala = $2 \mathrm{~cm}$. 

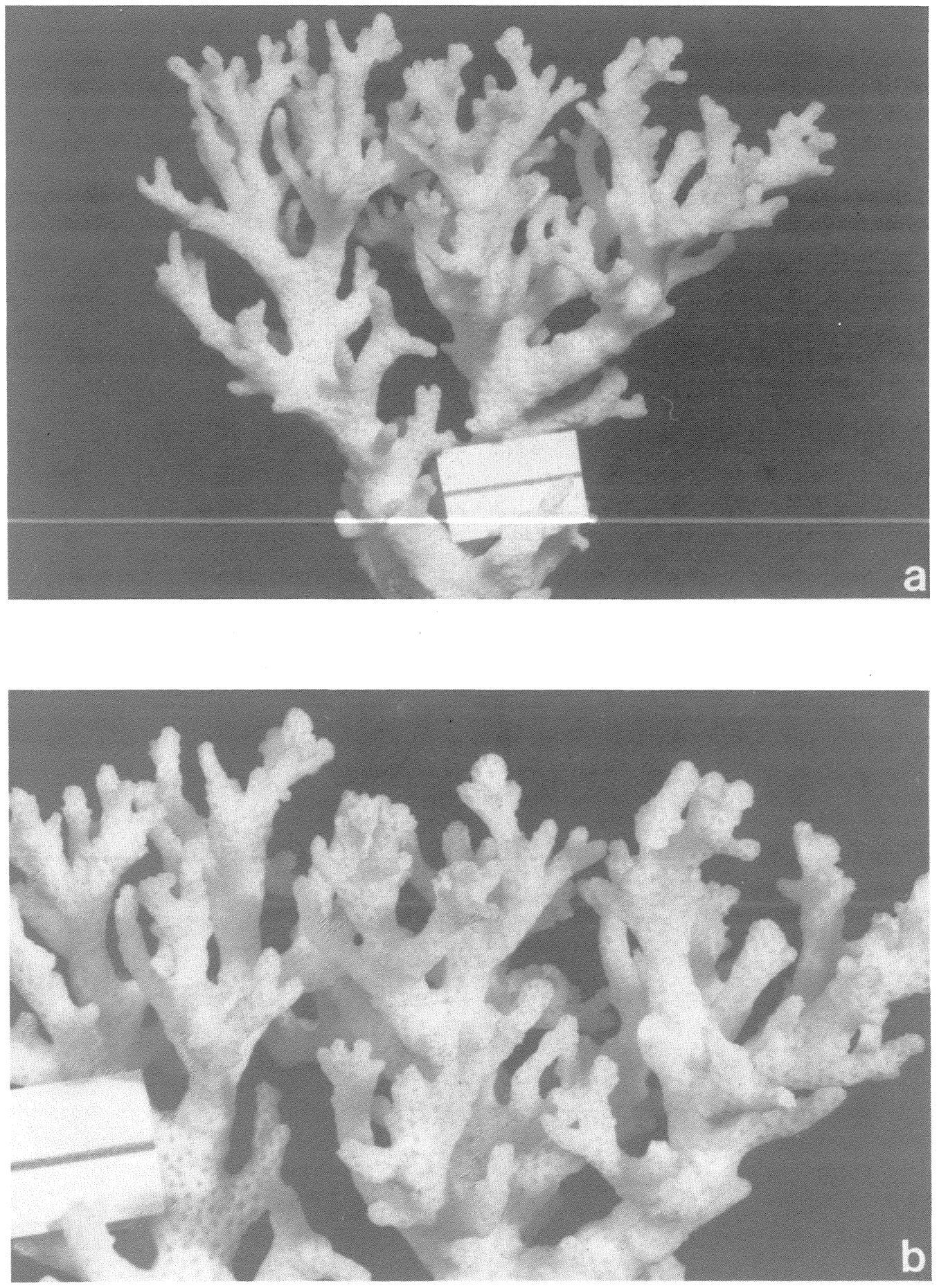

Fig. 3. a) Pocillopora damicornis (UCR 703), Sándalo, Golfo Dulce, $5 \mathrm{~m}, 8 . \mathrm{I} .1993$, escala $=2 \mathrm{~cm}$; b) detalle, escala $=1.2 \mathrm{~cm}$. 

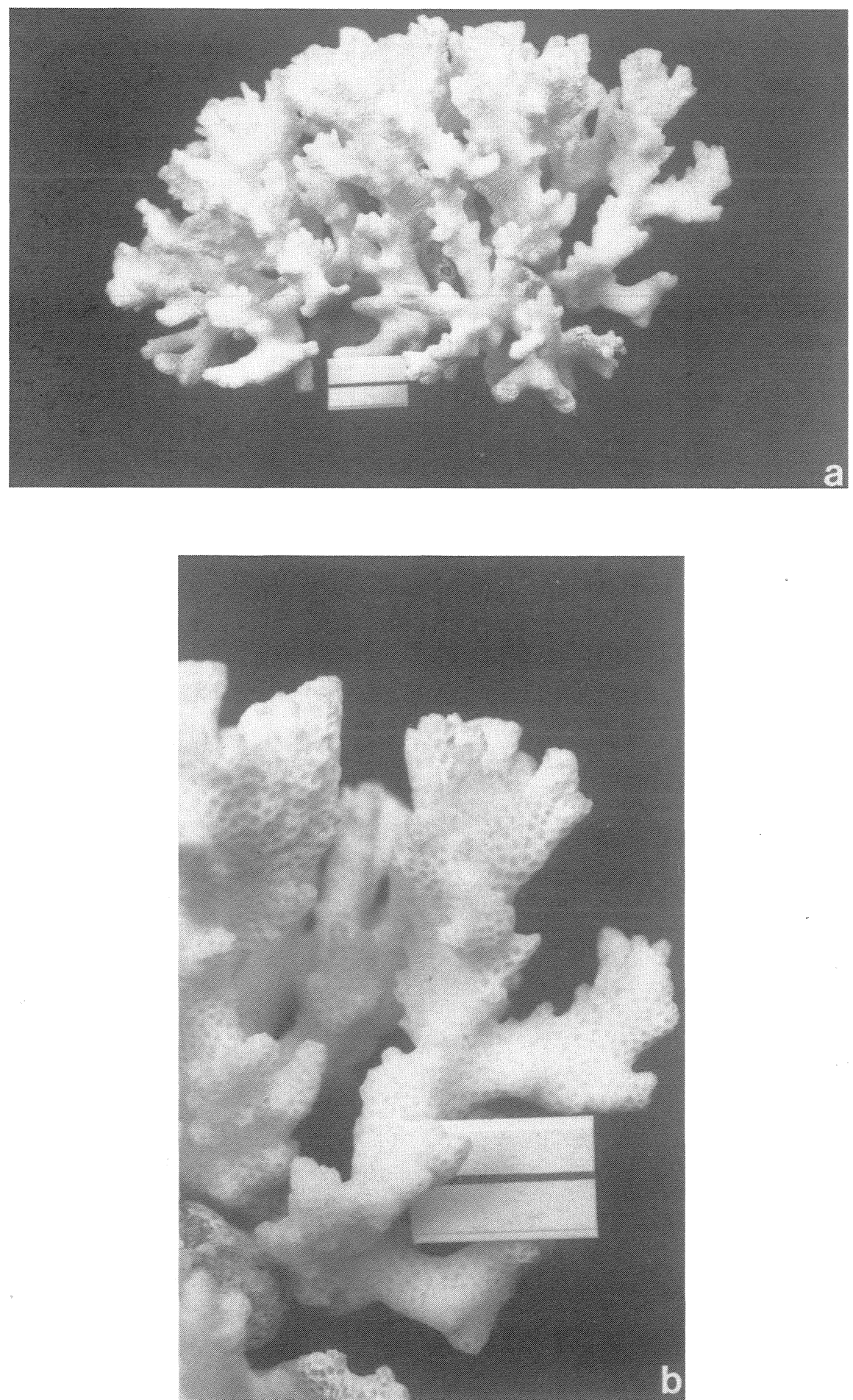

Fig. 4. a) Pocillopora elegans (UCR 748), Bajo Rojo, lado norte de la Península de Santa Elena, Guanacaste, 5 m, 7.I.1994, escala $=2 \mathrm{~cm}$; b) detalle, escala $=2 \mathrm{~cm}$. 

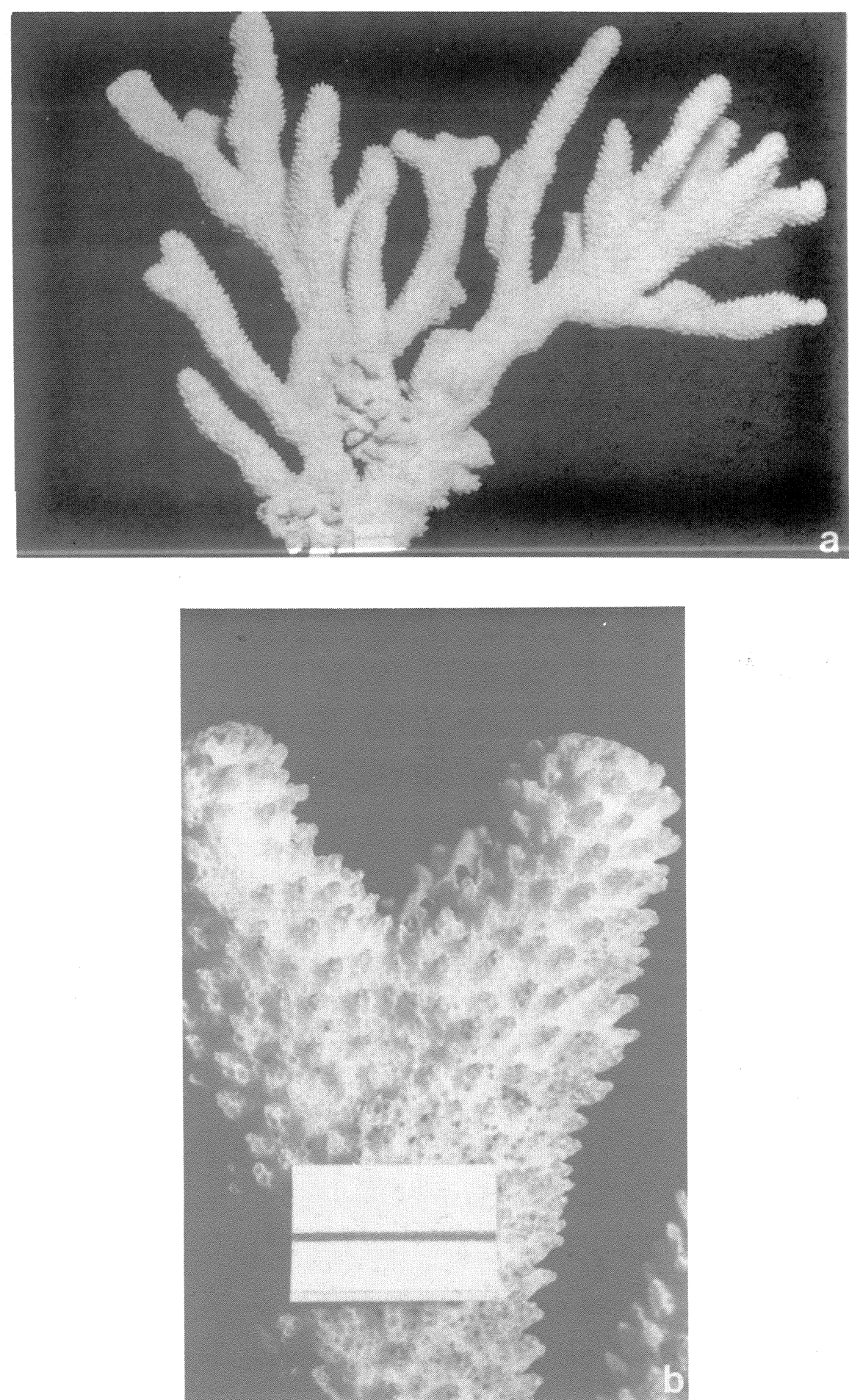

Fig. 5. a) Pocillopora eydouxi (UCR 44), Bahía Waffer, Isla del Coco, 1.IV.1975, escala $=2 \mathrm{~cm}$; b) detalle, escala $=1.8 \mathrm{~cm}$. 

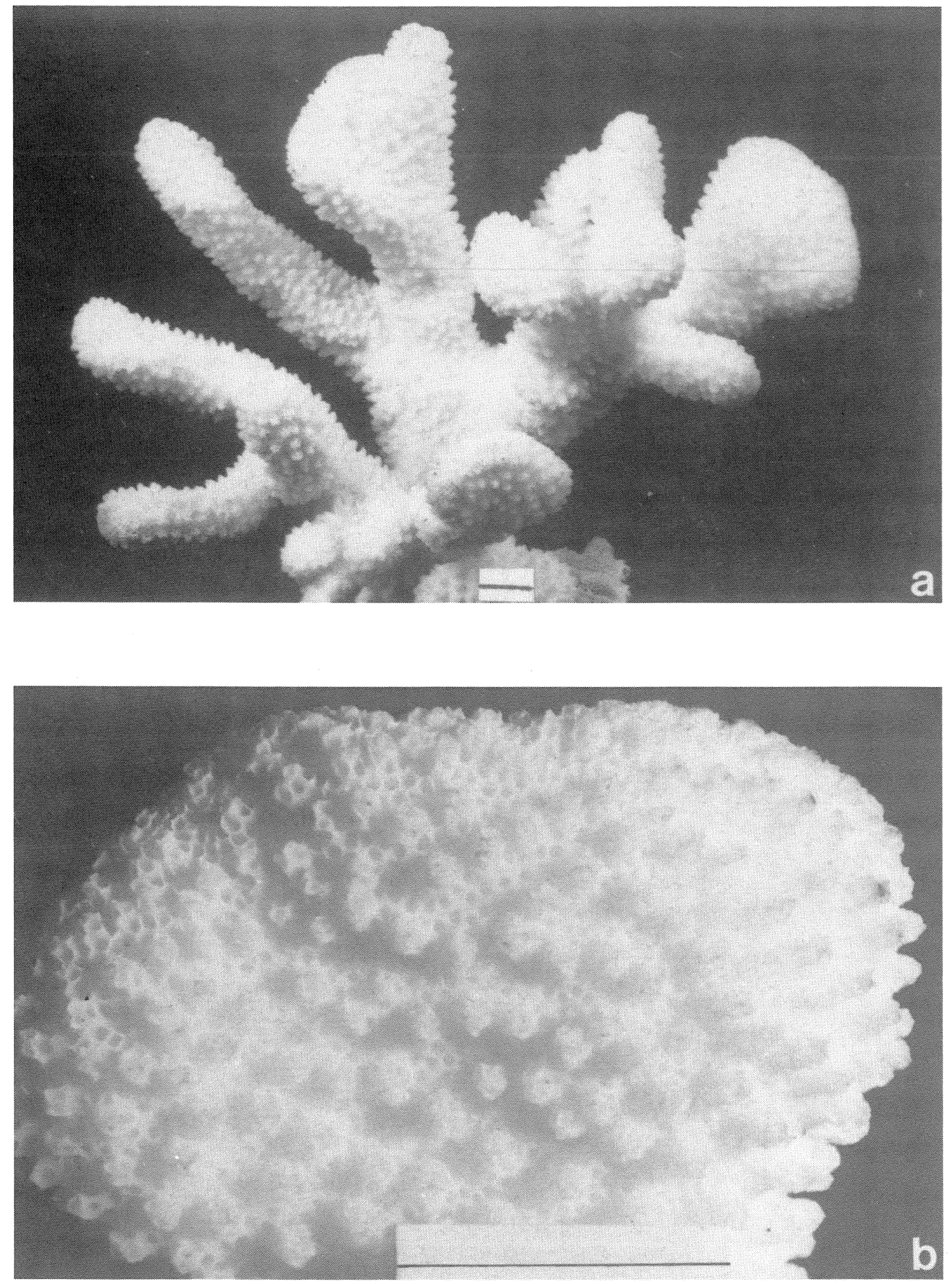

Fig. 6. a) Pocillopora meandrina (UCR 553), Punta Ballena, Isla del Caño, $4 \mathrm{~m}, 5$.VII.1988, escala $=2 \mathrm{~cm}$; b) detalle, escala $=2$ $\mathrm{cm}$. 

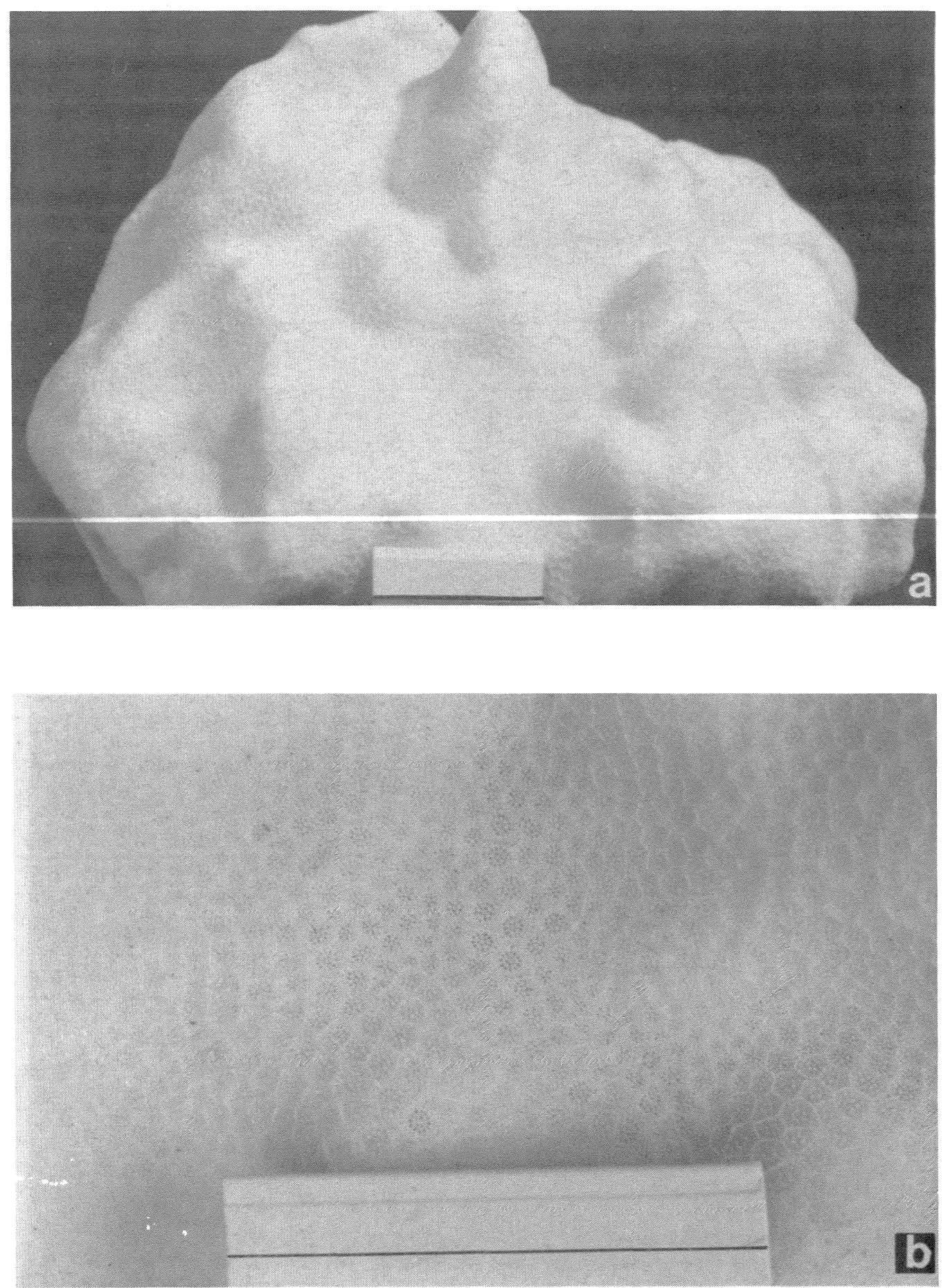

Fig. 7. a) Porites lobata (UCR 288), Platanillo, Isla del Caño, $6 \mathrm{~m}, 24 . \mathrm{I} .1984$, escala $=2 \mathrm{~cm}$; b) detalle, escala $=2 \mathrm{~cm}$. 


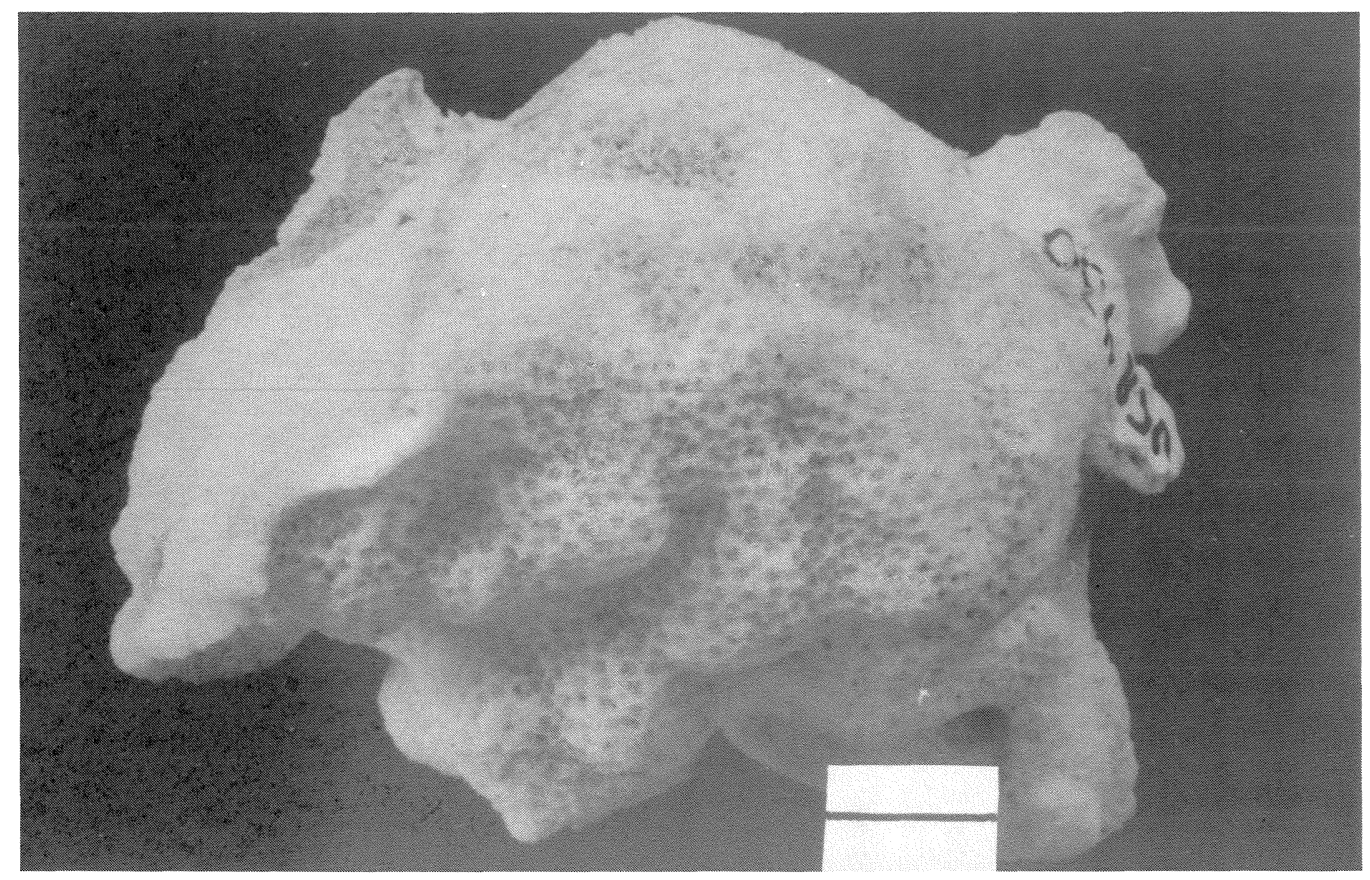

Fig. 8. Porites panamensis (UCR 430), Isla del Caño, $4 \mathrm{~m}, \mathrm{I} .1980$, escala $=2 \mathrm{~cm}$.

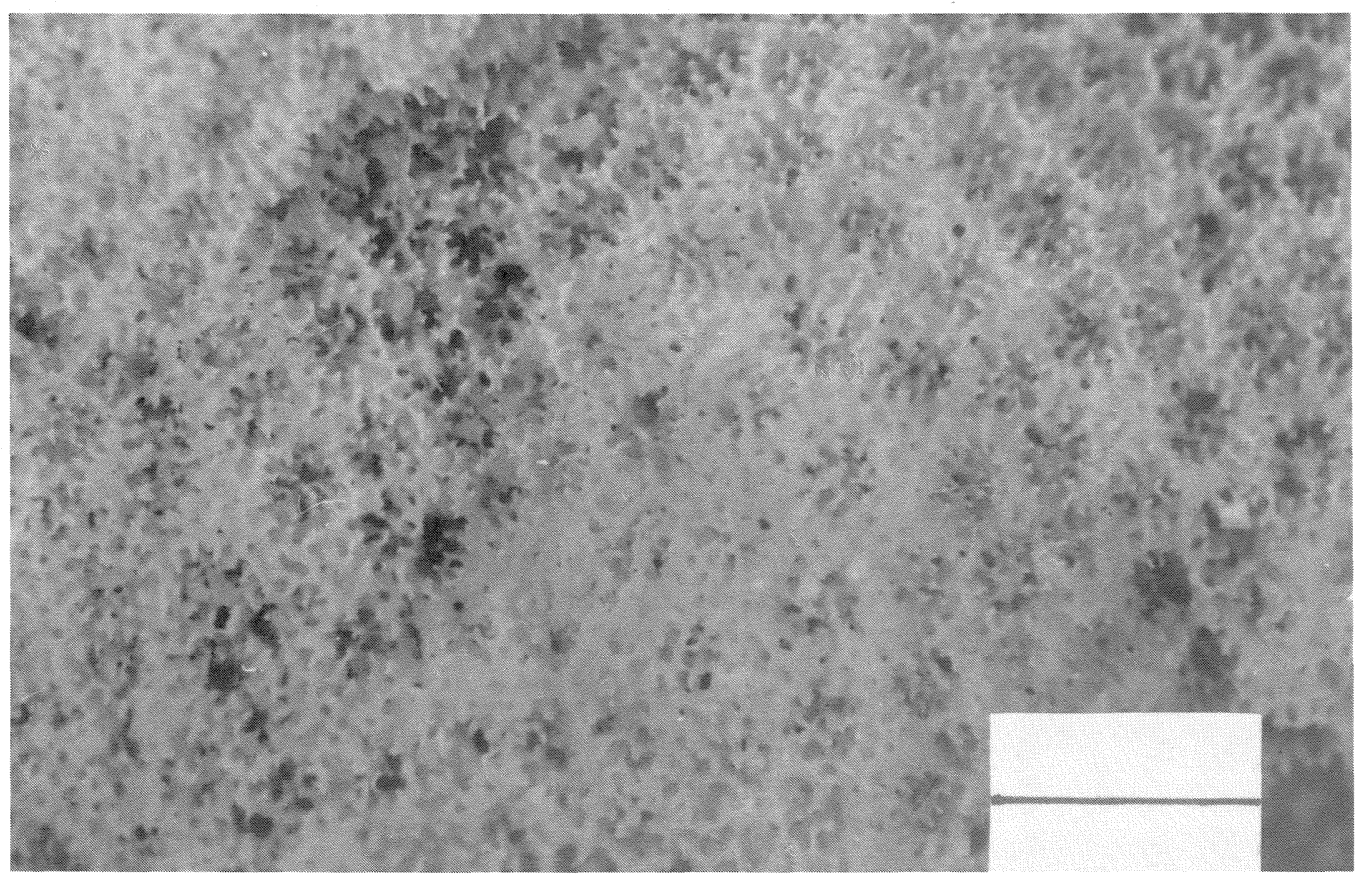

Fig. 9. Detalle de Porites (Synarea) rus (UCR 663), Sámara, Guanacaste, $7 \mathrm{~m}, 4$. VI.1983, escala $=0.5 \mathrm{~cm}$. 


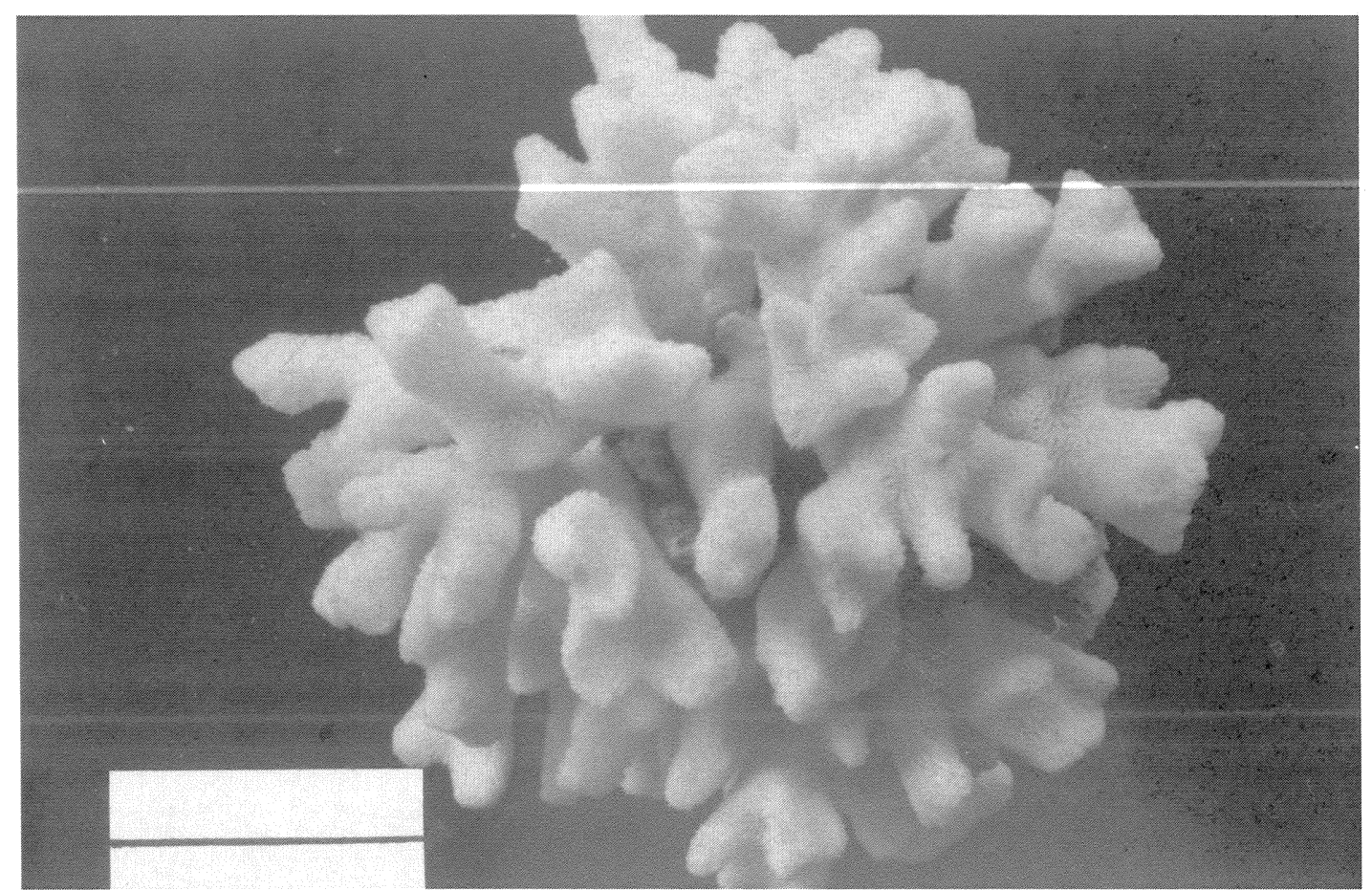

Fig. 10. Psammocora obtusangula (UCR 556), Punta El Bajo, Golfo Dulce, Puntarenas, 3 m, 21.IX.1988, escala = $0.75 \mathrm{~cm}$. 

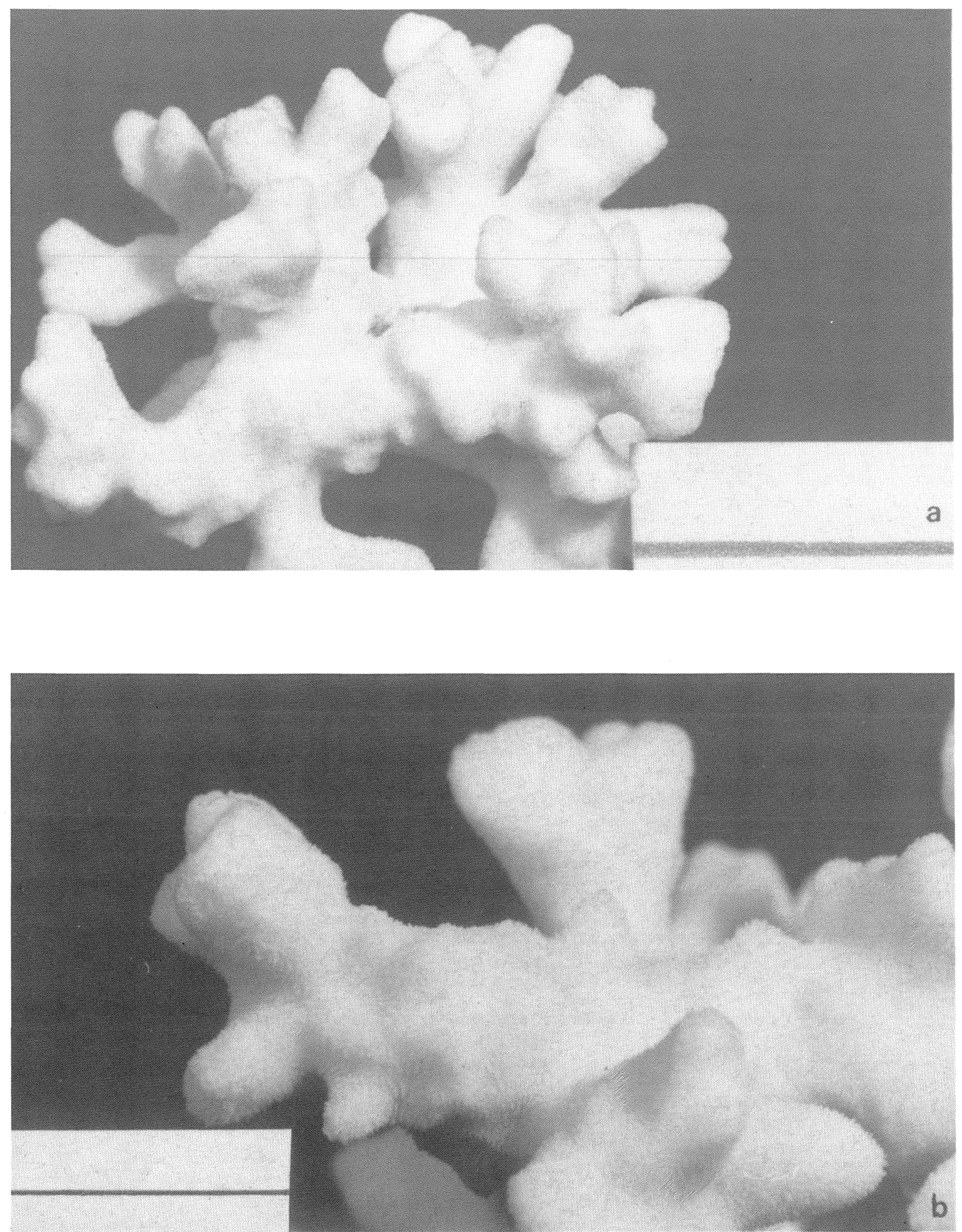

Fig. 11. a) Psammocora stellata (UCR 337), Playas del Coco, Guanacaste, $4 \mathrm{~m}, 8.1 .1984$, escala $=2 \mathrm{~cm}$; b) detalle, escala $=1 \mathrm{~cm}$. 

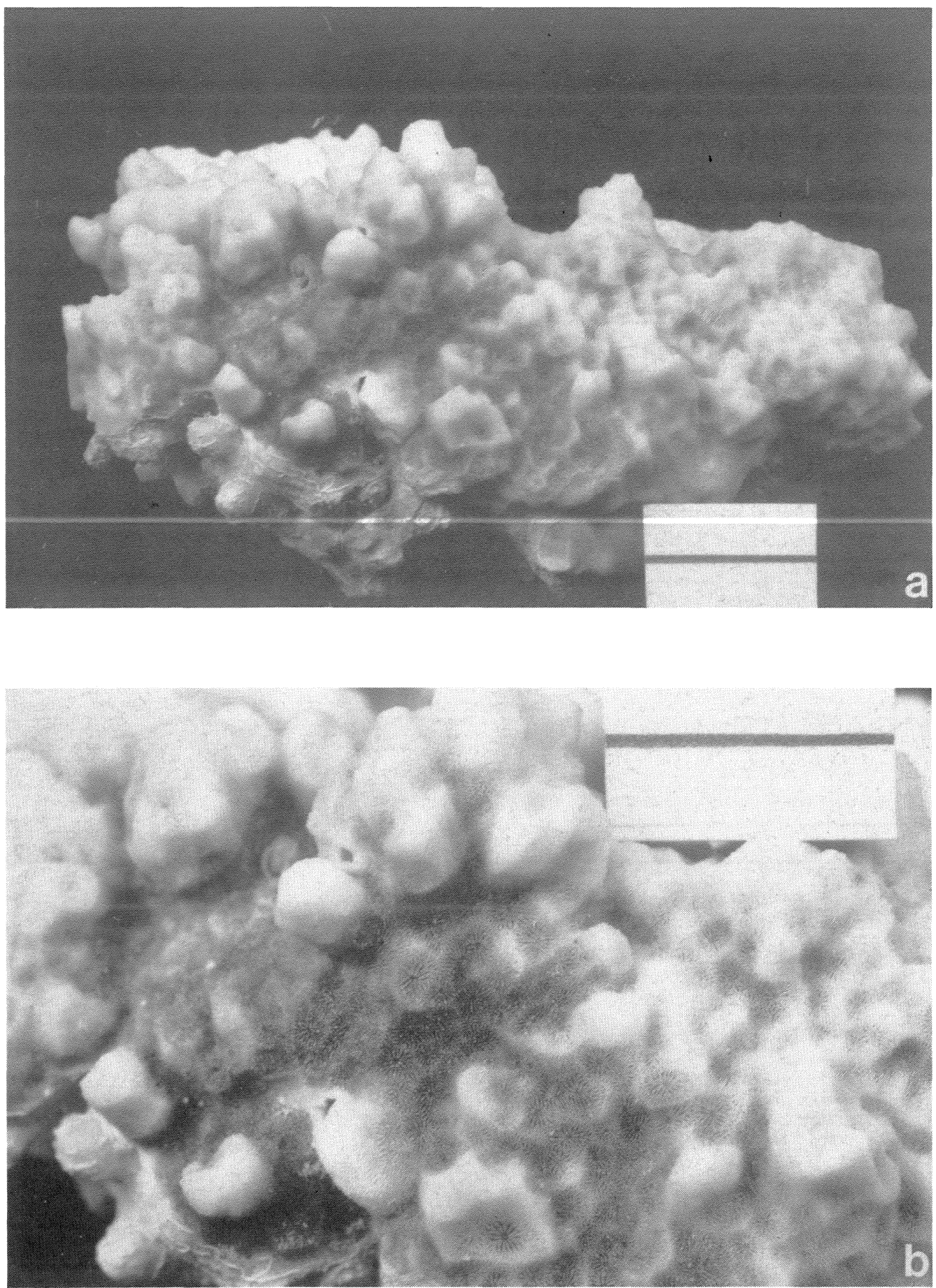

Fig. 12. a) Psammocora superficialis (UCR 719), Punta Presidio, Isla del Coco, $17 \mathrm{~m}, 9 . \mathrm{II} .1993$, escala $=2 \mathrm{~cm}$; b) detalle, escala $=2 \mathrm{~cm}$. 

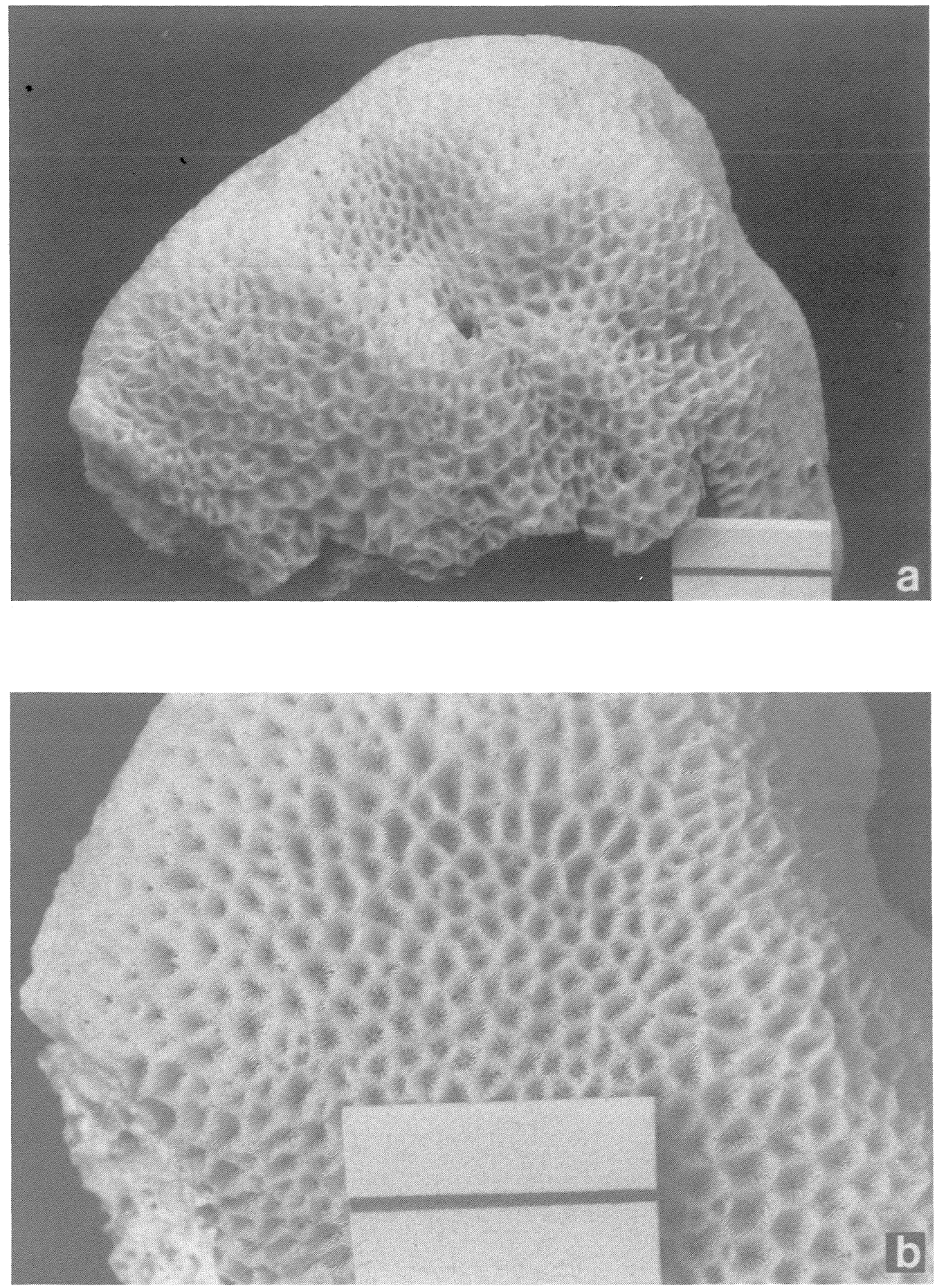

Fig. 13. a) Gardineroseris planulata (UCR 425), Isla del Caño, $6 \mathrm{~m}, \mathrm{I} .1980$, escala $=2 \mathrm{~cm}$; b) detalle, escala $=2 \mathrm{~cm}$. 


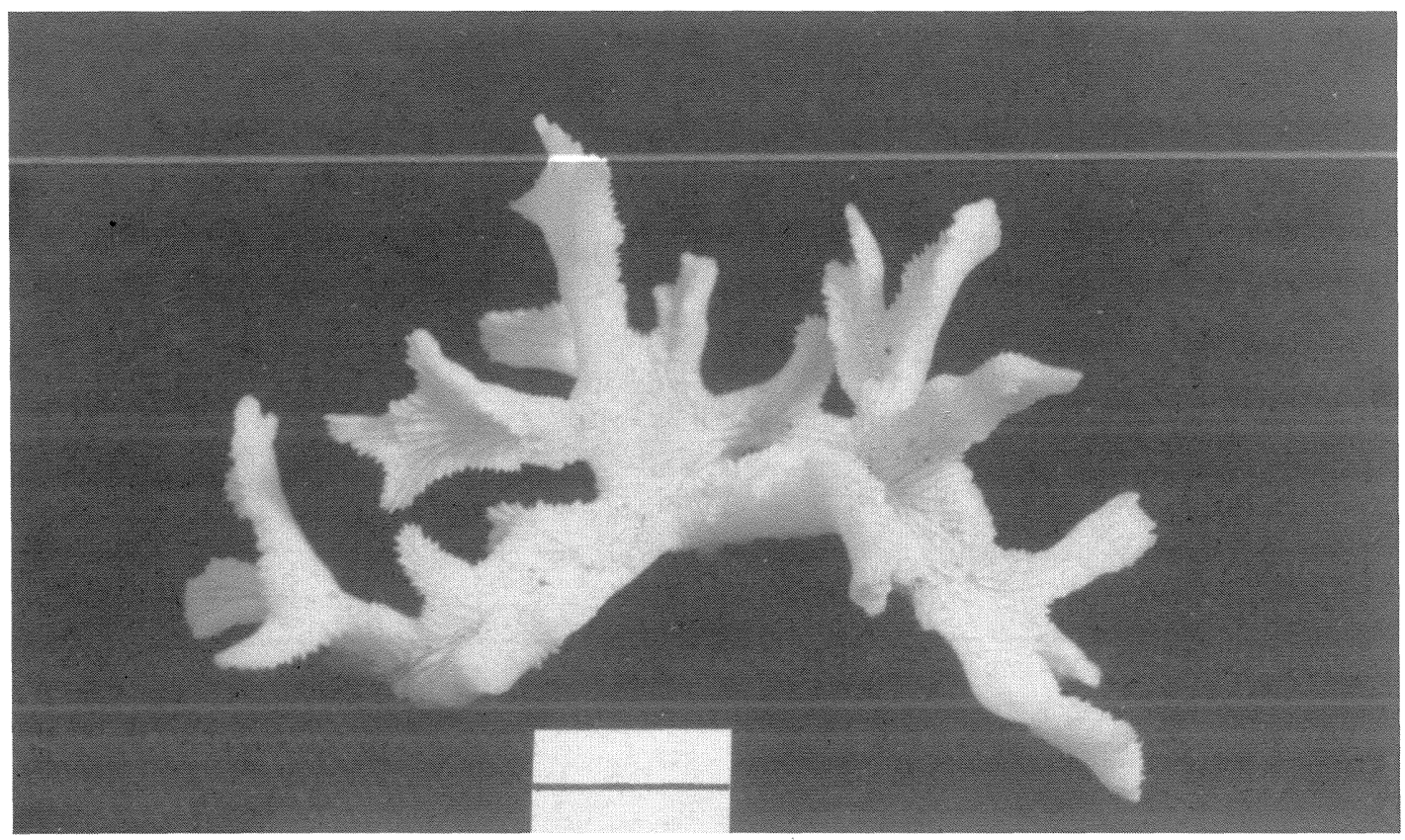

Fig. 14. Leptoseris papyracea (UCR 420), Playa Ocotal, Guanacaste, $10 \mathrm{~m}$, XII.1982, escala $=1 \mathrm{~cm}$. 

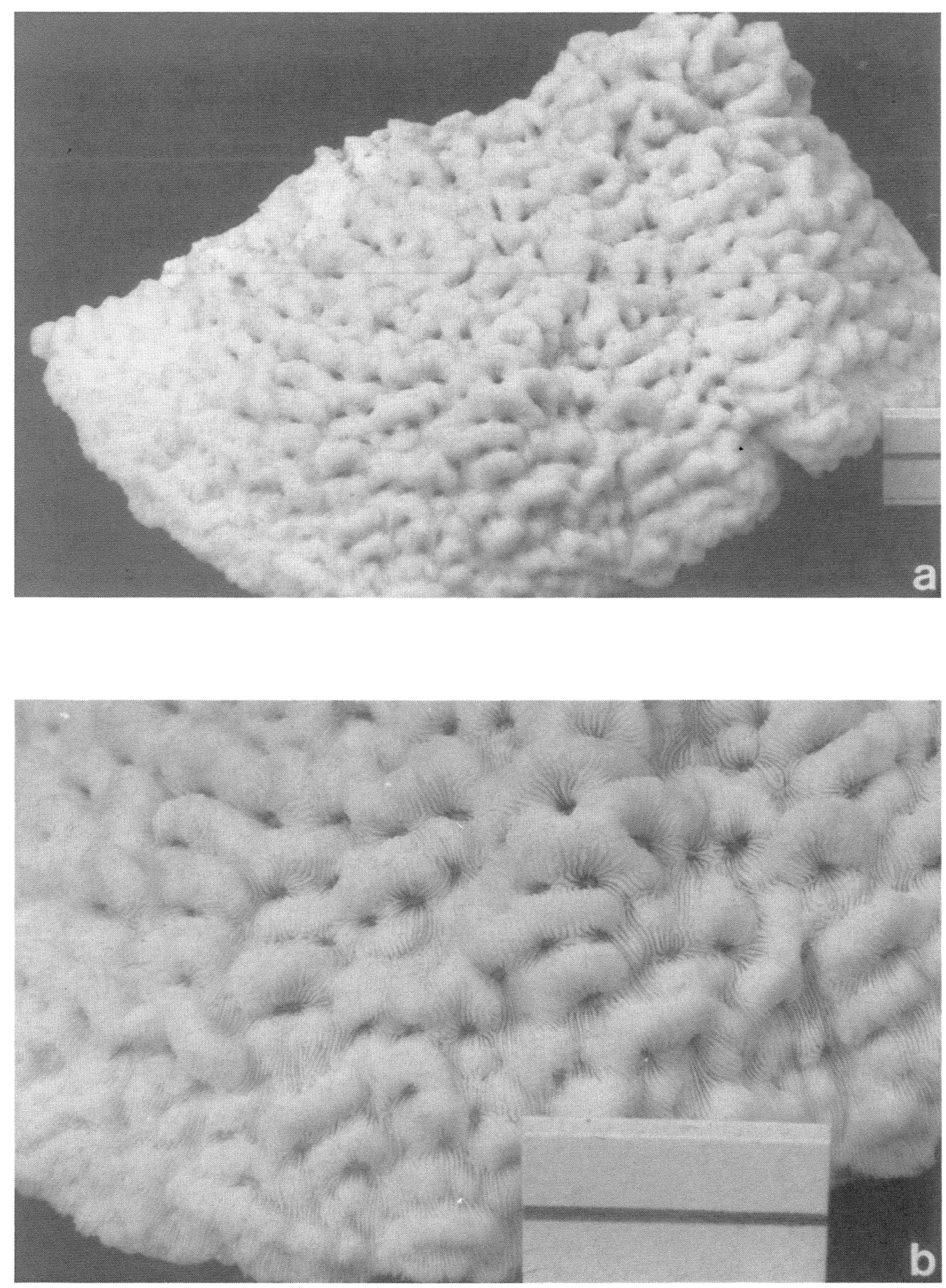

Fig. 15. a) Leptoseris scabra (UCR 715), Punta Presidio, Isla del Coco, $12 \mathrm{~m}, 9.1 \mathrm{II} .1993$, escala $=1 \mathrm{~cm}$; b) detalle, escala $=2 \mathrm{~cm}$. 

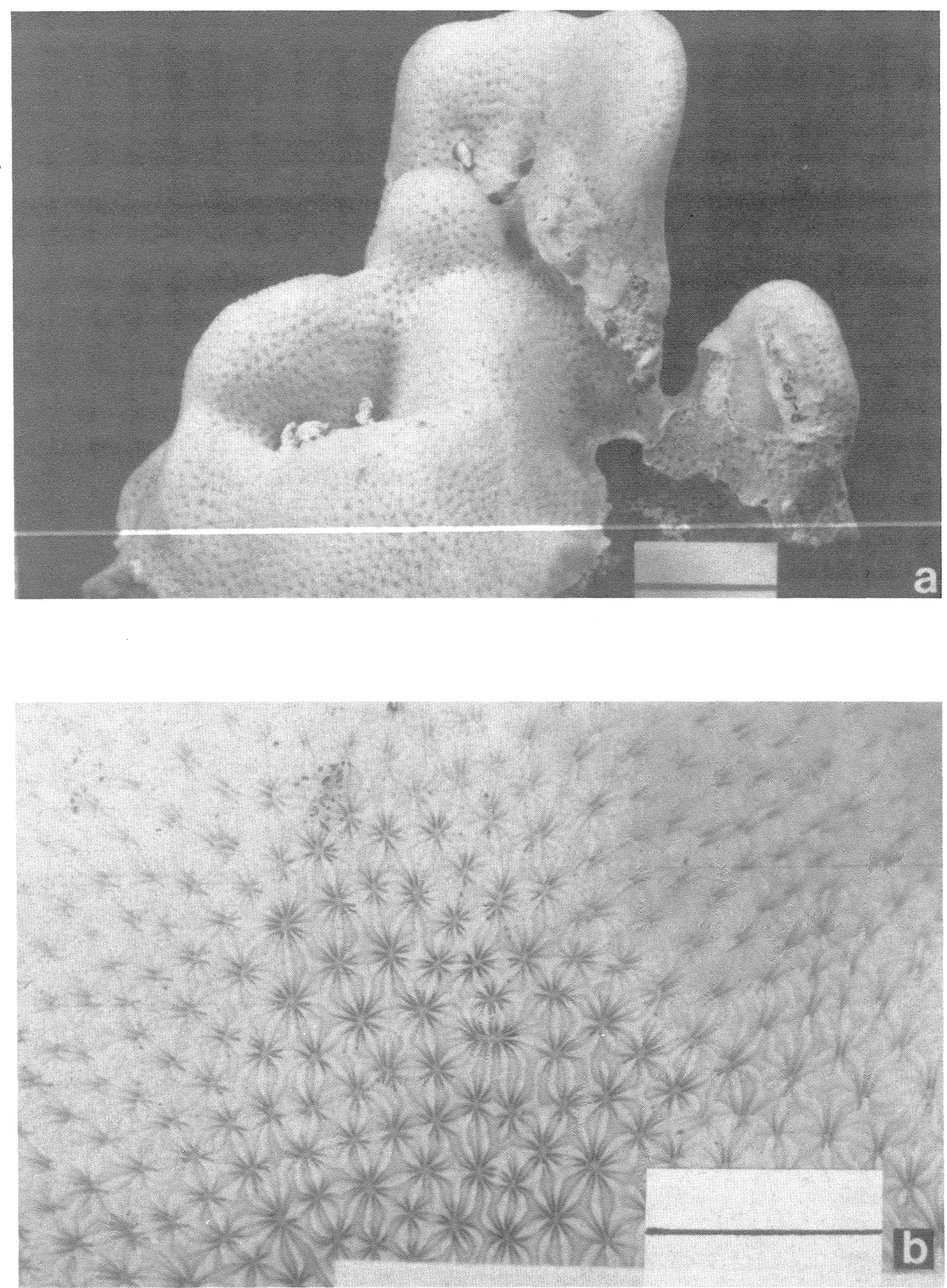

Fig. 16. a) Pavona clavus (UCR 321), Punta Mala, Puntarenas, $10 \mathrm{~m}, 24.1 \mathrm{I} .1984$, escala $=2 \mathrm{~cm}$; b) detalle, escala $=0.4 \mathrm{~cm}$. 

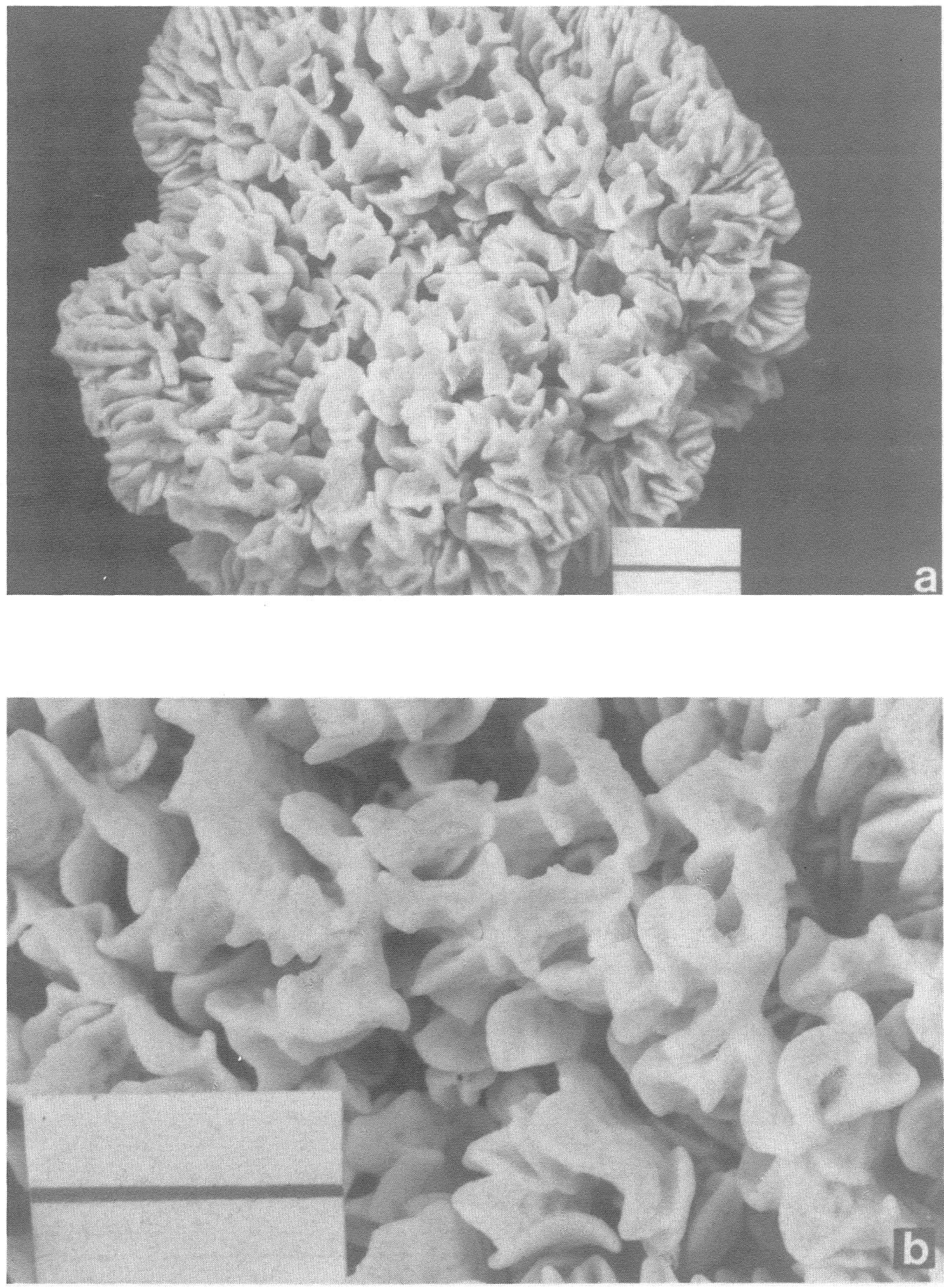

Fig. 17. a) Pavona frondifera (UCR 667), Estación Barracuda, Isla del Caño, $16 \mathrm{~m}, 3$. VI.1992; escala = $2 \mathrm{~cm}$; b) detalle, escala = $2 \mathrm{~cm}$. 

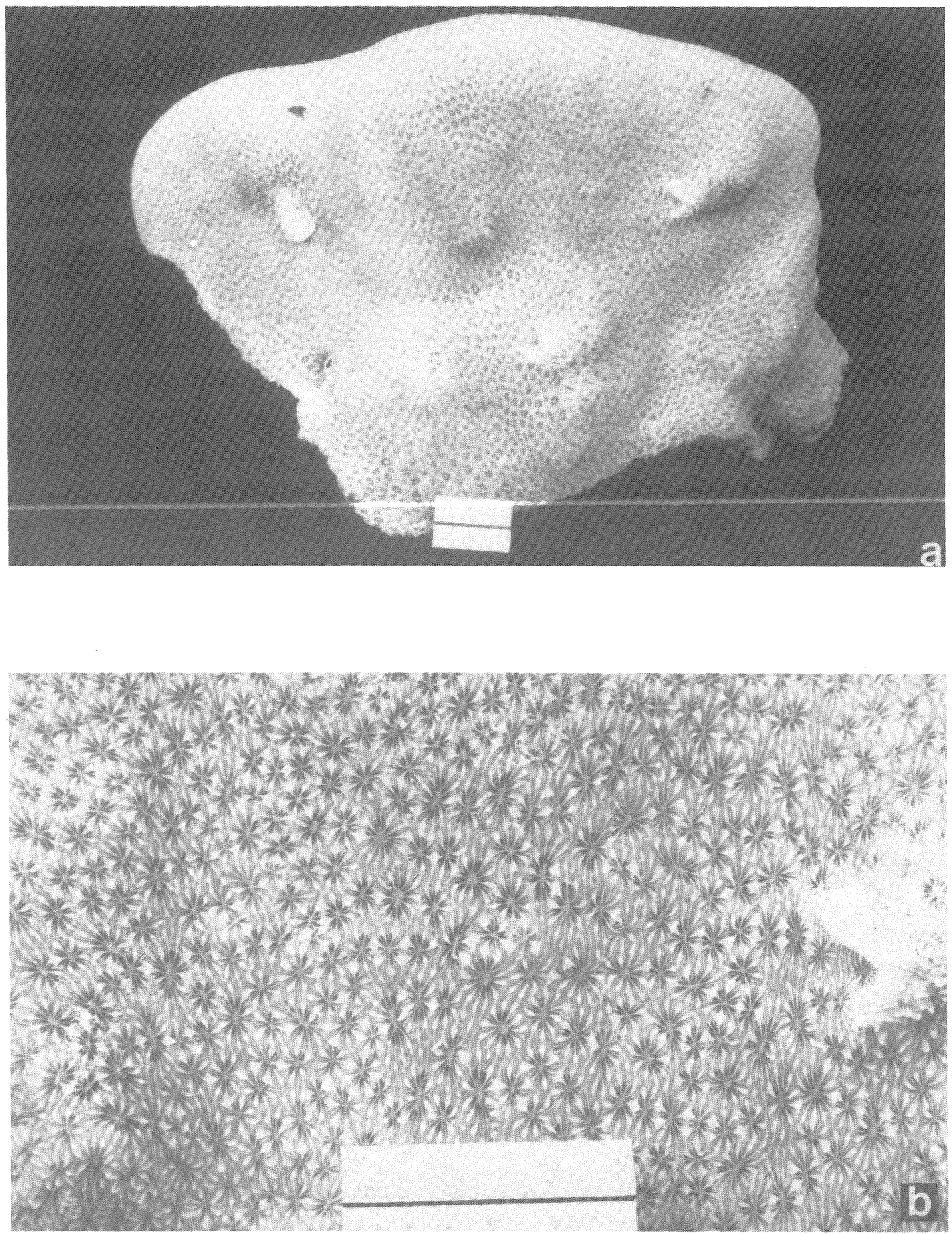

Fig. 18. a) Pavona gigantea (UCR 731), Punta Buena, Bahía Culebra, Guanacaste, $2 \mathrm{~m}, 25$. VI.1993, escala $=2 \mathrm{~cm}$; b) detalle, escala $=2 \mathrm{~cm}$; c) Pavona gigantea forma de profundidad (UCR 328), Playa Ocotal, Guanacaste, $16 \mathrm{~m}, 23.1 \mathrm{I} .1984$, escala = $2 \mathrm{~cm}$; d) detalle, escala $=2 \mathrm{~cm}$. 

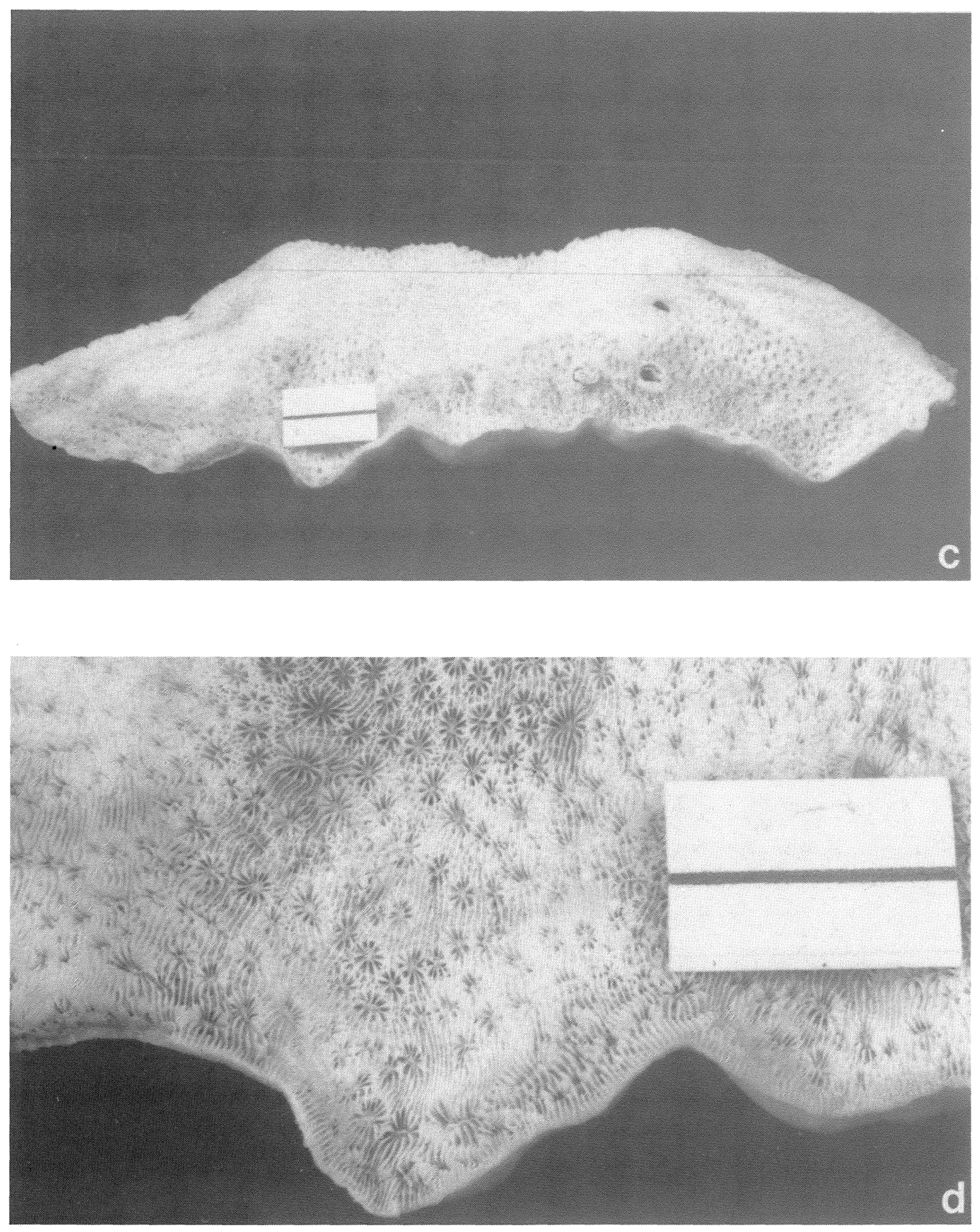

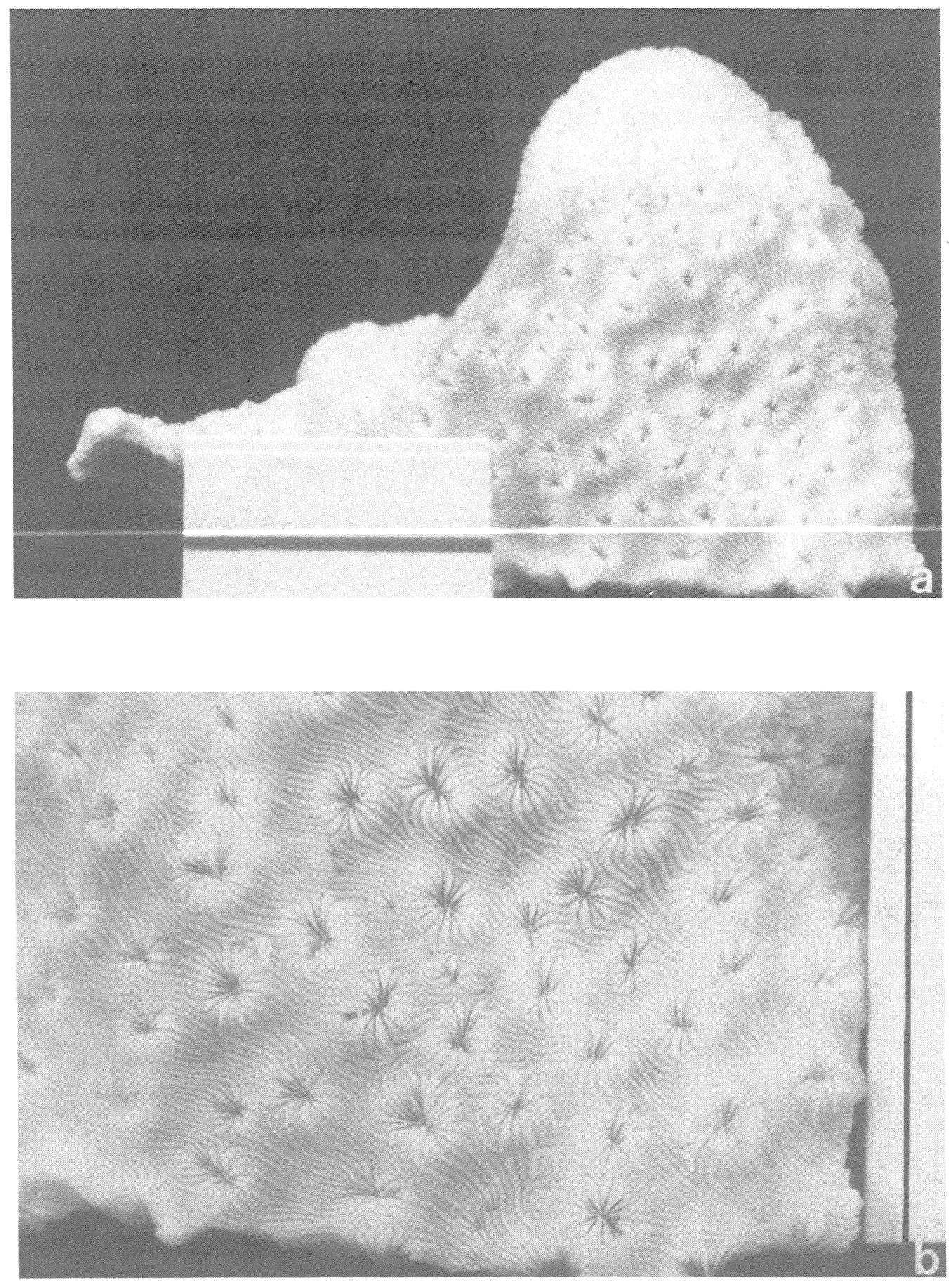

Fig. 19. a) Pavona maldivensis (UCR 710), Punta Presidio, Isla del Coco, $16 \mathrm{~m}, 9 . \mathrm{II} .1993$, escala $=2 \mathrm{~cm}$; b) detalle, escala $=1.2$ $\mathrm{cm}$. 

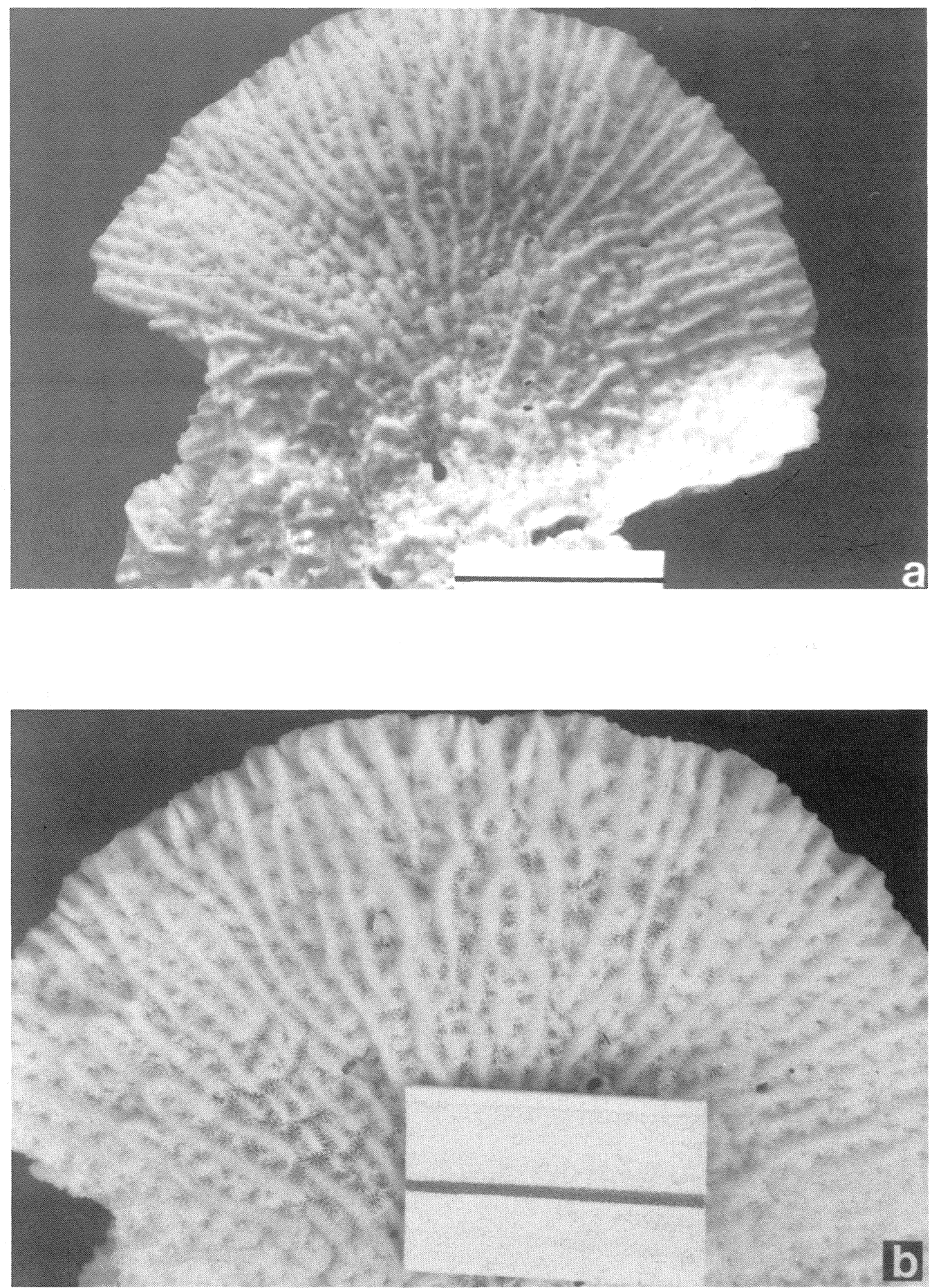

Fig. 20. a) Pavona varians (UCR 397), Isla del Caño, $13 \mathrm{~m}, 27$. VII.1985, escala $=2 \mathrm{~cm}$; b) detalle, escala $=2 \mathrm{~cm}$; c) Pavona varians (UCR 712), Punta Presidio, Isla del Coco, $16 \mathrm{~m}$, 9.II.1993, escala $=2 \mathrm{~cm} ; \mathrm{d}$ ) detalle, escala $=2 \mathrm{~cm} ; \mathrm{e}$ ) Pavona varians (UCR 685), Islas Pelonas, Bahía Culebra, Guanacaste, $19 \mathrm{~m}, 27$. VIII.1992, escala $=2 \mathrm{~cm} ; \mathrm{f}$ ) detalle, escala $=2 \mathrm{~cm}$. 

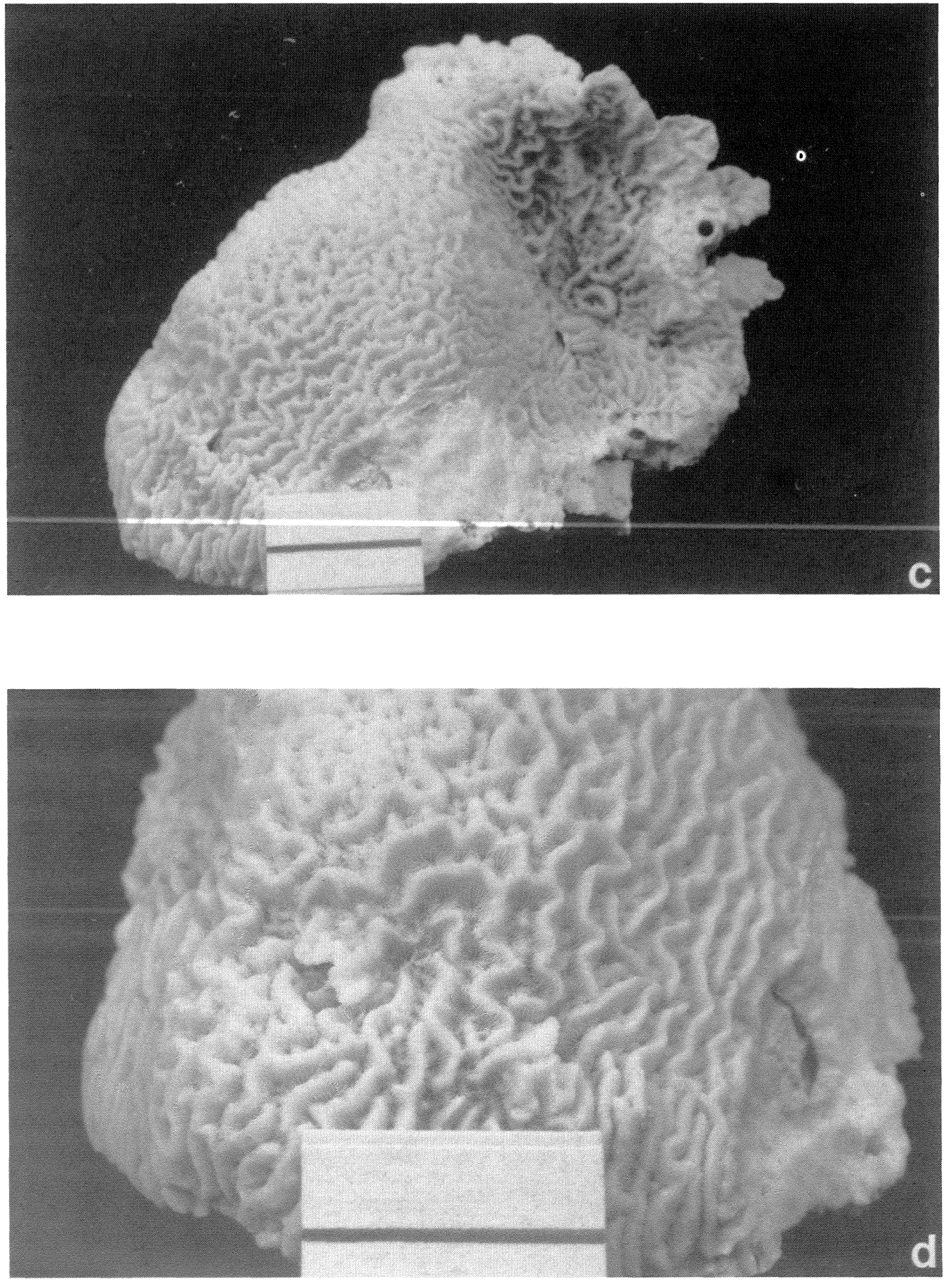

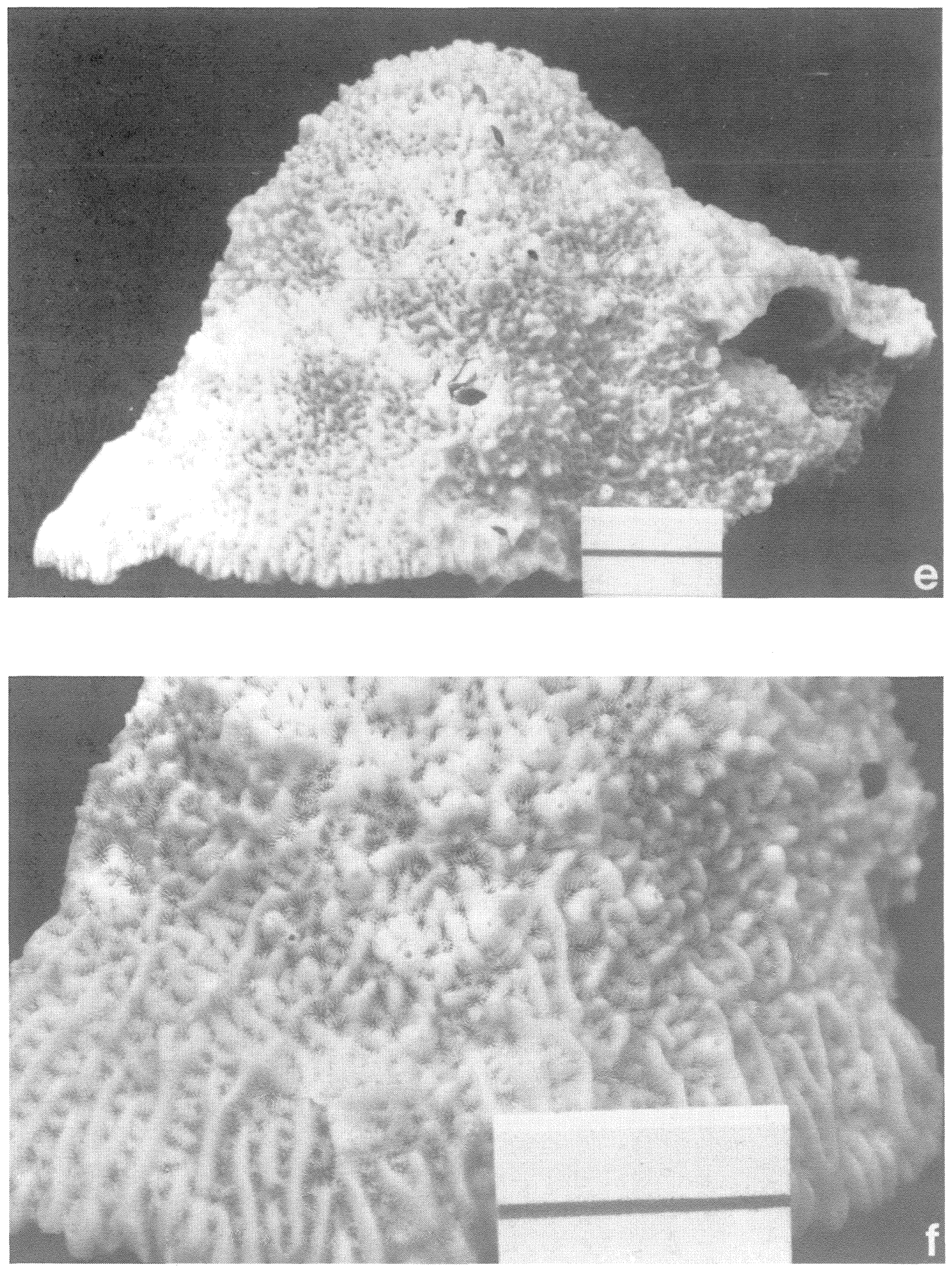

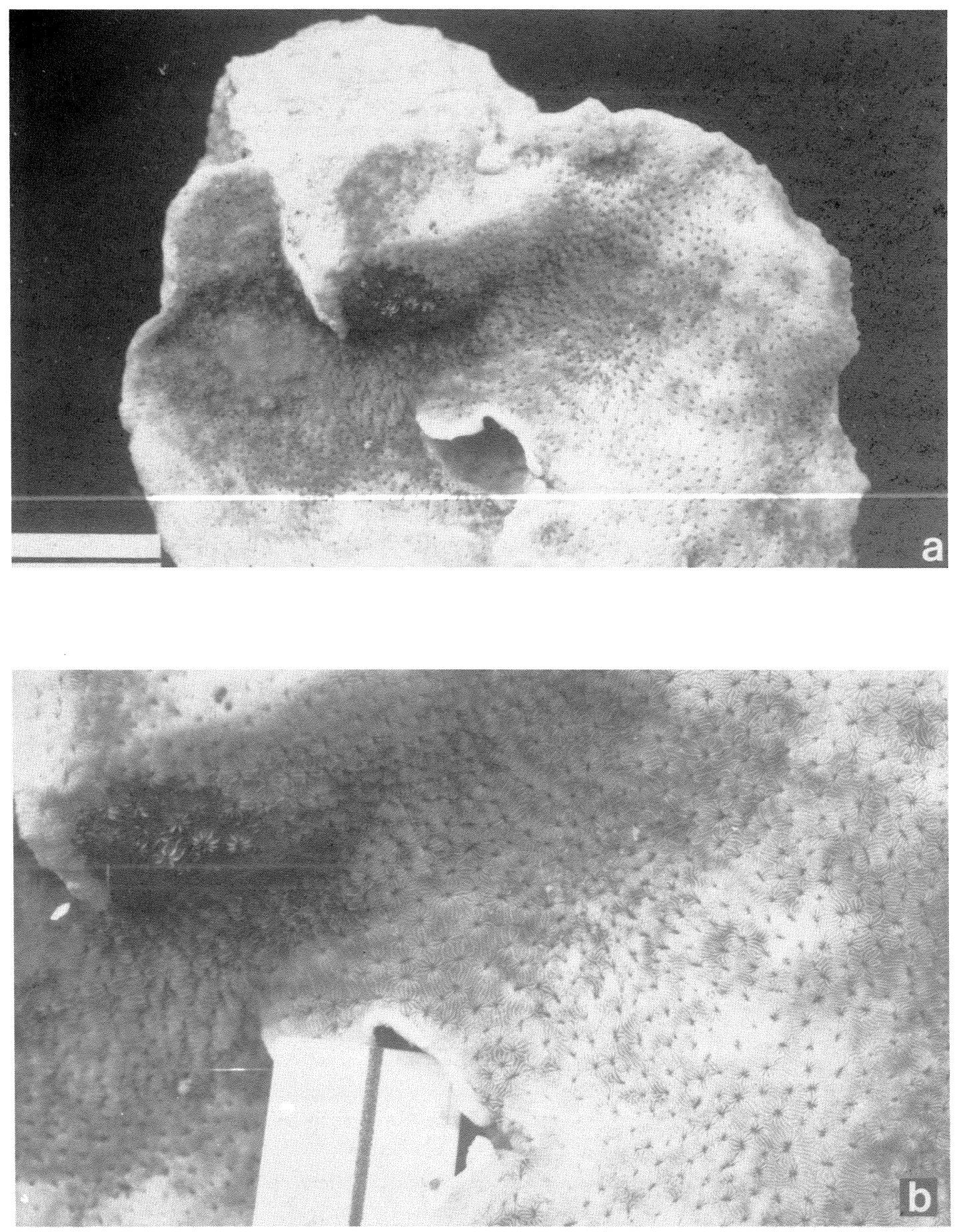

Fig. 21. a) Pavona xarifae (UCR 709), Punta Presidio, Isla del Coco, $19 \mathrm{~m}, 9.1 \mathrm{I} .1993$, escala $=2 \mathrm{~cm}$; b) detalle, escala $=1.5 \mathrm{~cm}$. 
12. Gardineroseris planulata (Dana, 1846)

\section{DESCRIPCION:}

Coral masivo con cálices profundos, de hasta $3 \mathrm{~mm}$, y de 3 a $5 \mathrm{~mm}$ de diámetro. Septos pequeños pero distintivos. Fig. 13 a, b.

\section{DISTRIBUCION:}

Costa Rica: Restringido al sur del país: Península de Osa, Isla del Caño, Isla del Coco.

Resto del mundo: Especie de distribución muy amplia, Mar Rojo, Océano Indico y el Océano Pacífico (Veron 1993).

\section{HISTORIA NATURAL:}

Gardineroseris planulata es una especie poco abundante en nuestros arrecifes. Su crecimiento es masivo y lento $(1 \mathrm{~cm} /$ año para Costa Rica: Guzmán \& Cortés 1989b). Actualmente se encuentran muy pocas colonias de tamaños superiores a los $50 \mathrm{~cm}$. Su reproducción es principalmente sexual (Glynn et al. 1996). La especie es depredada por Acanthaster planci, la cual podría serun factor importante en su limitada distribución, abundancia y tamaño.

\section{Leptoseris papyracea (Dana, 1846)}

\section{DESCRIPCION:}

Ramas muy delgadas (aproximadamente 5 $\mathrm{mm}$ ), ligeramente contorsionadas, con cálices de un solo lado, con septos bien definidos. Fig. 14 .

\section{DISTRIBUCIONGEOGRAFICA:}

Costa Rica: Bahía Culebra, Isla del Coco.

Resto del mund•: Desde Madagascar hasta el Pacífico Americano(Dinesen 1980, Veron 1993).
14. Leptoseris scabra Vaughan, 1907

\section{DESCRIPCION:}

Esqueleto grueso, incrustante, con cálices prominentes de un solo lado. Fig. 15 a, b.

\section{DISTRIBUCIONGEOGRAFICA:}

\section{Costa Rica: Isla del Coco.}

Resto del mundo: Especie de distribución amplia, Mar Rojo, Océano Indico y Océano Pacífico (Dinesen 1980, Veron 1993).

\section{HISTORIA NATURAL DE LAS ESPECIES DEL GENERO Leptoseris:}

Leptoseris papyracea se conocía para Costa Rica por solo unos pocos especímenes recolectados en 1983 en Playa Ocotal. Desde entonces y hasta 1996 no se había vuelto a observar, cuando en Punta Esmeralda, Bahía Culebra, Carlos E. Jiménez encontró un arrecife construido por esta especie. No se conocía la existencia previa de arrecifes construidos por esta especie, ya que en otras regiones del mundo es poco abundante (Dinesen 1980). El arrecife de Punta Esmeralda cubre un área aproximada de $2500 \mathrm{~m}^{2}$ y se extiende desde los $8 \mathrm{~m}$ a los $12 \mathrm{~m}$ de profundidad. Huecos hechos en la estructura y pruebas con varillas de construcción, indican un espesor mínimo de $0.4 \mathrm{~m}$ (C.E. Jiménez com. pers. 1996).

Leptoseris scabra es poco abundante y solo ha sido recolectada en la Isla del Coco. Vive en forma incrustante en grietas en las rocas. Este es un nuevo registro para el Pacífico Oriental.

\section{Pavona clavus (Dana, 1846)}

\section{DESCRIPCION}

Colonias hemisféricas o lobuladas, de tamaños variables: de unos pocos centímetros a $10 \mathrm{~m}$ de 
diámetro en arrecifes en Guanacaste. Cálices de 2,5 a 3,5 mm de diámetro, septos bien definidos y dentados. Fig. 16 a, b.

\section{DISTRIBUCIONGEOGRAFICA:}

Costa Rica: Archipiélago de las Islas Murciélago, Bahía Culebra, Manuel Antonio, Península de Osa, Golfo Dulce, Isla del Caño, Isla del Coco.

Resto del mundo: Especie de distribución amplia, Golfo Pérsico (Sheppard \& Sheppard 1991), y desde el Mar Rojo hasta la costa de América Central (Veron 1993).

\section{Pavona frondifera (Lamarck, 1816)}

\section{DESCRIPCION:}

Colonias incrustantes o hemisféricas, con proyecciones pronunciadas del esqueleto parecidas a hojas; desde colonias pequeñas incrustantes de unos pocos centímetros, hasta colonias hemisféricas de $1 \mathrm{~m}$ de diámetro. Fig. 17 a, b.

\section{DISTRIBUCIONGEOGRAFICA:}

Costa Rica: Bahía Culebra, Golfo Dulce, Isla del Caño.

Resto del mundo: Desde la costa este de Africa a la costa Pacífica de América Central (Veron 1993).

\section{Pavona gigantea Verrill, 1869}

\section{DESCRIPCION:}

Colonias masivas, con cálices grandes $(>1$ $\mathrm{mm}$ ) y generalmente conextenciones en forma de alerosenla parte inferior, especialmente a mayores profundidades. Fig. 18 a, b, c, d.

\section{DISTRIBUCIONGEOGRAFICA:}

Costa Rica: Area de Conservación Guancaste, Bahía Culebra, Punta Mala, Manuel Antonio, Península de Osa, Golfo Dulce, Isla del Caño, Isla del Coco.

Resto del mundo: Pacífico Oriental y la Isla Fanning (Wells 1983).

\section{Pavona maldivensis (Gardiner, 1905)}

\section{DESCRIPCION:}

Colonias hemisféricas, generalmente pequeñas, con los cálices de 1,5 a $2,0 \mathrm{~mm}$ y elevados 1 a 2 mm, con 16 a 20 septos. Fig. 19 a, b.

\section{DISTRIBUCION GEOGRAFICA:}

CostaRica:Areade Conservación Guanacaste, Bahía Culebra, Parque Marino Ballena, Isla del Caño, Isla del Coco.

Resto del mundo: Especie de distribución muy amplia, del Mar Rojo y la costa este de Africa hasta la costa de América Central (Veron 1993).

\section{Pavona varians Verrill, 1864}

\section{DESCRIPCION:}

Colonias incrustantes, de cálices pequeños $(<$ $1 \mathrm{~mm}$ ), casi rellenos por los septos y columnela. El envés es liso y a veces con ondulaciones que corresponden a ondulaciones en la superficie superior. Esta especie muestra un amplio rango de variación; algunas de las cuales posiblemente sean especies nuevas (P.W. Glynn com. pers. 1997). Fig. 20 a, b, c, d, e, f. 


\section{DISTRIBUCIONGEOGRAFICA:}

Costa Rica: Area de Conservación Guancaste, Bahía Culebra, Punta Mala, Manuel Antonio, Península de Osa, Golfo Dulce, Isla del Caño, Isla del Coco.

Resto del mundo: Especie de distribución amplia desde el Mar Rojo y la costa este de Africa hasta el Pacífico americano (Veron 1993).

20. Pavona xarifae Scheer \& Pillai, 1974

\section{DESCRIPCION:}

Colonias incrustantes con cálices pequeños, menos de $1 \mathrm{~mm}$, alineados; aproximadamente 10 cálices por $\mathrm{cm}^{2}$. El corallum parece una "oreja de res" (Scheer \& Pillai 1974). De 16 a 18 septos generalmente, pero a veces hasta 24 , alternando su tamaño. Los septos se extienden de un cálice a otro. Columnela poco desarrollada. Fig. 21 a, b.

\section{DISTRIBUCION GEOGRAFICA:}

Costa Rica: Isla del Coco.

Resto del mundo: Desde las Islas Nicobaren el Oceáno Indico hasta las Islas Cook en el Océano Pacífico (Scheer \& Pillai 1974; Veron 1993).

\section{COMENTARIO:}

Este es un nuevo registro para el Pacífico Oriental.

\section{HISTORIA NATURAL DE LAS ESPECIES DEL GENERO Pavona:}

Las especies más abundantes de este género y de las que se conoce más son: Pavona clavus, $P$. gigantea y $P$. varians. Estas especies son de amplia distribución en el arrecife, excepto en plataformas someras y de alta energía. Generalmente forman parches con individuos de diversos tamaños. $P$. gigantea pueden alcanzar más de un metro de alto y formar parches de varioscientos de metroscuadrados (Cortés 19961997a). P. clavus crece normalmente en colonias de menos de un metro, pero en Guancaste se han encontrado colonias de hasta $10 \mathrm{~m}$ de diámetro (Cortés \& Jiménez en prep.). En otras regiones del Pacífico Oriental se conoce de colonias de $P$. clavus de hasta 5 metros de altura (Colgan 1990). El crecimiento de estas dos especies oscila entre 0.5 y $1.2 \mathrm{~cm} /$ año; $P$. varians es de forma incrustante y de un crecimiento inferior a $0.5 \mathrm{~cm} /$ año (Guzmán $\&$ Cortés 1989b). Sinembargo, esta últimaespecie pareciera tener una mayor capacidad para colonizar espacios libres que las otras especies. La reproducción de $P$. varians es sexual lo cual facilita su reclutamiento. Las tres especies son presa preferida de Acanthaster planci, la cual a veces limita su abundancia. La capacidad de regeneración de estas especies es lenta, y generalmente las heridas por peces balístidos se transforman en necrosis del tejido, que son aprovechadas por los peces damiselas para expandir sus territorios.

Dentro del género Pavona es posible que haya más especies que las descritas aquí. Por ejemplo, la revisión de Wells (1983) indica la presencia de tresespecies en el Pacífico oriental.En los últimos años se han observado (o diferenciado otras): maldivensis que se incluía dentro de gigantea y frondifera dentro de varians. En este trabajo indicamos otra especie no informada anteriormente, xarifae, y bajo varians posiblemente estamos incluyendo varias especies (P.W. Glynn com. pers. 1997). Estudios morfométricos y genéticos más detallados son necesarios para aclarar la taxonomía de estas especies.

\section{Fungia (Cycloseris) curvata (Verrill, 1870)}

\section{DESCRIPCION:}

Esqueleto de un solo pólipo, relativamente grueso y denso, difícil de quebrar, y arqueado. Vive despegado del sustrato. Hay varios ciclos de 
septos de diferentes tamaños, ornamentados con pequeños dientes. Ilustradoen Hoeksema (1989).

\section{DISTRIBUCIONGEOGRAFICA:}

Costa Rica: Bahía Culebra, Golfo Dulce, Isla del Coco.

Resto del mundo: Mar Rojo, Zanzíbar, Golfo Pérsico, Filipinas, Indonesia, Papua Nueva Guinea, Islas Galápagos, México (Hoeksema (1989); Japón (Nishihira \& Veron 1995).

\section{Fungia(Cycloseris) distorta Michelin, 1842}

\section{DESCRIPCION:}

Esqueleto de un solo pólipo, frágil, fácil de quebrar, generalmente confisuras, y relativamente planas. Viven separados del sustrato. Septos abundantes en varios ciclos y cubiertos por gránulos. Ilustrado en Hoeksema (1989).

\section{DISTRIBUCION GEOGRAFICA:}

Costa Rica: Bahía Culebra, Isla del Coco.

Resto del mundo: Mar Rojo (Sheppard \& Sheppard 1991), Zanzíbar, Mozambique, Maldivas, Filipinas, Indonesia, Japón, Papua Nueva Guinea, Palau, Islas Marshall, Hawaii, México (Hoeksema 1989).

\section{HISTORIA NATURAL DE LAS ESPECIES DEL GENERO Fungia:}

Fungia (Cycloseris) curvata fue descubierta viva recientemente (1996) porCarlos E. Jiménez, en Bahía Culebra. Había sido recolectada por primera vezenCostaRicacercadePuerto Jiménez, Golfo Dulce (especímenes en el Museo de Historia Natural, Smithsonian Institution, USNM 77890, no se cuenta con otros datos), posteriormente se recolectó en la Isla del Coco en 1986, en aguas profundas (USNM 80083, 80084).
Fragmentos muertos en forma de cuña de Fungia (Cycloseris) distorta se encuentran en forma abundante en Bahía Culebra, Guanacaste. Actualmente la especies que se encuentra viva allíes $F$. $(C$.) curvata, y se ha podido observar su forma de fragmentación y regeneración. Esta especiesecomportasimilara Fungia (Cycloseris) distorta (informada como Diaseris distorta) en Japón. En algún momento de su vida se empieza a disolver el esqueleto a lo largo de líneas longitudinales, hasta que se separa en forma de cuñas (Yamashiro \& Nishihira 1994). Posteriormente, cada cuña empieza a regenerarel resto del cálice (Yamashiro 1992).

\section{AGRADECIMIENTOS}

Se le agradece a la Vicerrectoría de Investigación de laUniversidadde CostaRica(Proyectos 808-83-135, 808-92-237, 808-96-601), a CONICIT (Proyecto 90-326-BID), a Peter W. Glynn (Proyecto U.S. National Science Foundation OCE 8415615), y al Smithsonian Tropical Research Institute (STRI Research Opportunity a HMG) por la ayuda económicaque hizo posible las recolectas de corales y esta publicación. Las mayoría de las fotografías se hicieron en STRI y se contó con la ayuda de Marco Guerra y Antonio Montaner, otras las tomó Guillermo Vargas de la Unidad de Microscopía Electrónica, Universidad de Costa Rica; Gerardo Chávez ayudó en el ordenamiento de las fotografías; y Rita Vargas con el mapa. Esta es una contribución conjunta del Museo de Zoología, Escuela de Biología, y del Centro de Investigación en Ciencias del Mary Limnología, ambos de la Universidad de Costa Rica.

\section{RESUMEN}

Se describen veinte y dos especies de corales ecleractinios. zooxantelados del Pacífico de Costa Rica se describen. Se incluyen claves para los géneros y especies. Se presenta su distribución geográfica en Costa Rica y en todo el mundo, así como aspectos de la historia general de las especies. Dieciséis 
especies tiene distribuciones muy amplias, desdeen Mar Rojo o el Océano Indico hasta la costa Pacífica de América, dos especies son endémicas del Pacífico Oriental, y cuatro especies se encuentran solo en Océano Pacífico; no hay ninguna especie en común con el Caribe-Atlántico. Dos especies, Leptoseris scabra y Pavona xarifae, son registros nuevos para el Pacífico Oriental.

\section{REFERENCIAS}

Bakus, G. J. 1975. Marine zonation and ecology of Cocos Island, off Central America. Atoll Res. Bull. 179: 1-9.

Birkeland, C., D. L. Meyer, J.P. Stames \& C. L. Buford. 1975. Subtidal communities of Malpelo Island. In The Biological Investigation of Malpelo Island, Colombia, J. B. Graham (ed.). Smithson. Cont. Zool. 176: 55-68.

Colgan, M.W. 1990. El Niño and the history of eastern Pacific reef building, p. 183-229. In Glynn PW (ed), Global ecological consequences of the 1982-83El Niño-Southern Oscillation. Elsevier, Amsterdam.

Cortés, J. 1986. Biogeografía de corales hermatípicos: el istmo Centro Americano. Anales Inst. Cien. Mar Limnol., UNAM 13: 297-304.

Cortés, J. 1990. The coral reefs of Golfo Dulce, Costa Rica: distribution and community structure. Atoll Res. Bull. 334: 1-37.

Cortés, J. 1996-1997a. Comunidades coralinas y arrecifes del Area de Conservación Guanacaste, Costa Rica. Rev. Biol. Trop. 44(3)/45(1): 623-625.

Cortés, J. 1996-1997b. Biodiversidad marina de Costa Rica: Filo Cnidaria. Rev. Biol. Trop. 44(3)/45(1): 323-334.

Cortés, J. \& C.E. Jiménez. 1996. Coastal-marineenvironments of Parque Nacional Corcovado, Puntarenas, Costa Rica. Rev. Biol. Trop. 44, Suppl. 3: 35-40.

Cortés, J. \& M.M. Murillo. 1985. Comunidades coralinas y arrecifes del Pacífico de Costa Rica. Rev. Biol. Trop. 33 197-202.

Cortés, J., I.G. Macintyre \& P.W. Glynn. 1994. Holocene growth history of an eastern Pacific fringing reef, Punta Islotes, Costa Rica. Coral Reefs 13: 65-73

Devaney, D.M. \& J.C.Lang. 1987. Scleractinia (stony corals) of Enewetak Atoll, p. 67-76. In D.M. Devaney, E.S Reese, B.L. Burch \& P. Helfrich (eds.), The Natural History of Enewetak Atoll, Vol. II Biogeography abd Systematics. U.S Dept. Energy, Washington, D.C.
Dinesen, Z. D. 1980. A revision of the coral genus Leptoseris (Scleractinia: Fungiina: Agariciidae). Mem. Qd. Mus. 20 181-235.

Dubois, R. \& M. Hatziolos. 1982. Inventario de recursos marinos: sin paginar. In Centro Científico Tropical (ed.), El Parque Nacional Manuel Antonio, Inventario Biológico Terrestre y Marino, Informe Final de Consultoría.

Durham, J.W. 1962. Corals from the Galápagos and Cocos Islands. Proc. Calif. Acad. Sci. 4th Ser. XXXII(2): 41-56.

Durham, J.W. 1966. Coelenterates, especialy stony corals from the Galápagos and Cocos Islands, p. 123-135. In R.I Bowman (ed.), The Galápagos. University of California, Berkeley, Los Angeles.

Durham, J.W. \& J.L. Barnard. 1952. Stony corals of the eastern Pacific collected by Velero III and Velero IV. Allan Hancock Pac. Exped. 16: 1-110.

Glynn, P.W. 1983. Crustacean symbionts and the defense of corals: coevolution on the reef?, p. 111-178. In M.H. Nitecki (ed.), Coevolution. University of Chicago, Chicago.

Glynn, P.W. 1997. Eastern Pacific reef coral biogeography and faunal flux: Durham's dilemma revisited. Proc. 8th Int. Coral Reef Symp., Panamá 1:371-378.

Glynn, P.W. \& G.M. Wellington. 1983. Corals and Corals Reefs of the Galápagos Islands. University of California, Berkeley. 330 p.

Glynn, P.W., E.M. Druffel \& R.B. Dunbar. 1983. A dead Central American coral reef tract: posible link with the Little Ice Age. J. Mar. Res. 41: 605-637.

Glynn, P.W., J.E.N. Veron \& G.M. Wellington. 1996 Clipperton Atoll (eastern Pacific): oceanography, geomorphology, reef-building coral ecology and biogeography. Coral Reefs 15: 71-99.

Glynn, P.W., S.B. Colley, N.J. Gassman, K. Black, J. Cortés \& J.L. Maté. 1996. Reef coral reproduction in the eastern Pacific: Costa Rica, Panama, and Galapagos Islands (Ecuador) - III. Agariciidae (Pavona gigantea and Gardineroseris planulata). Mar. Biol. 125: 579-601

Glynn, P.W., N.J. Gassman, C.M. Eakin, J. Cortés, D.B Smith \& H.M. Guzmán. 1991. Reef coral reproduction in the eastern Pacific: Costa Rica, Panama, and Galapagos Islands (Ecuador), Part I-Pocilloporidae. Mar. Biol. 109: 355-368. 
Glynn, P.W., S.B. Colley, C.M. Eakin, D.B. Smith, J. Cortés, N.J. Gassman, H.M. Guzmán, J.B. del Rosario \& J. Feingold. 1994. Reef coral reproduction in the eastern Pacific: Costa Rica, Panama, and Galapagos Islands (Ecuador) - 11. Poritidae. Mar. Biol. 118: 191-208.

Guzmán, H.M. 1986. Estructura de la comunidad arrecifal de la Isla del Caño, Costa Rica, y el efecto de perturbaciones natuales severas. Tesis de Maestría, Universidad de Costa Rica, San José, Costa Rica. 179 p.

Guzmán, H.M. 1988. Distribución y abundancia de organismos coralívoros en los arrecifes coralinos de la Isla del Caño, Costa Rica. Rev. Biol. Trop. 36: 191-207.

Guzmán, H.M. \& J. Cortés. 1989a. Coral reef community structure at Cañọ Island, Pacific Costa Rica. P.S.Z.N.I: Mar. Ecol. 10: 23-41.

Guzmán, H.M. \& J. Cortés. 1989b. Growth rates of eight species of scleractinian corals in the eastern Pacific (Costa Rica). Bull. Mar. Sci. 44: 1186-1194.

Guzmán, H. M. \& J. Cortés. 1992. Cocos Island (Pacific of Costa Rica) coral reefs after the 1982-83 El Niño disturbance. Rev. Biol. Trop. 40: 309-324.

Guzmán, H.M. \& J. Cortés. 1993. Los arrecifes coralinos del Pacífico Oriental Ecuatorial: Revisión y perspectivas. Rev. Biol. Trop. 41: 535-557.

Guzmán, H.M. \& J.D. López. 1991. Diet of the corallivorous pufferfish Arothron meleagris (Pisces: Tetradontidae) at Gorgona Island, Colombia. Rev. Biol. Trop. 39: 203-206.

Guzmán, H.M. \& D.R. Robertson. 1989. Population and feeding responses of the corallivorous pufferfishArothron meleagris to coral mortality in the eastern Pacific. Mar. Ecol. Prog. Ser. 55: 121-131.

Hertlein, L.G. 1963. Contribution to the biogeography of Cocos Island, including a bibliography. Proc. Calif. Acad. Sci. 4th Ser., XXXII(8): 219-289.

Hoeksema, B.W. 1989. Taxonomy, phylogeny and biogeography of mushroom corals (Scleractinia: Fungiidae). Zool. Verh. Leiden 254: 1-295.

Hodgson, G. \& K. Carpenter. 1995. Scleractinian corals of Kuwait. Pac. Sci. 49: 227-246.

Macintyre, I.G., P.W. Glynn \& J. Cortés. 1992. Holocene reef history in the eastern Pacific: mainland Costa Rica, Caño Island, Cocos Island, and Galápagos Islands. Proc. 7th Int. Coral Reef Symp., Guam 2: 1174-1184.
Maragos, J.E. 1977. Order Scleractinia, stony corals. In D.M. Devaney \& L.G. Eldrege (eds.), Reef and Shore Fauna of Hawaii, Section 1: Protozoa through Ctenophora. B.P. Bishop Museum Spec. Publ. 64: 158-241.

Nemenzo, F. 1986. Guide to Philippine Flora and Fauna, Volume V: Corals. Natural Resources Management Center, Ministry of Natural Resources and University of the Philippines, Manila. 273 p.

Nishihira, M. \& J.E.N. Veron. 1995. Hermatypic corals of Japan. Kaiyusha, Tokio. 440 p.

Pichon, M. 1985. Scleractinia. In G. Richard (ed.), Récifs coralliens de Polynésie Française. Proc. 5th Int. Coral Reef Cong., Tahiti 2: 390-403.

Prahl, H. von \& H. Erhardt. 1985. Colombia, Corales y Arrecifes Coralinos. Presencia, Bogotá, Colombia. 325 p.

Randall, R.H. \& R.F. Myers. 1983. Guide to the coastal resources of Guam: Vol. 2, the corals. University of Guam, Mangilao, Guam. 290 p.

Richmond, R.H. 1985. Variations in the population biology of Pocillopora damicornis across the Pacific Ocean. Proc. 5th Int. Coral Reef Cong., Tahiti 6: 101-106.

Scheer, G. \& C.S.G. Pillai. 1983. Report on the stony corals from the Red Sea. Zoologica 133: 1-198.

Scott, P.B. 1984. The Corals of Hong Kong. Hong Kong University, Hong Kong. 112 p.

Sheppard, C.R.C. \& A.L.S. Sheppard. 1991. Corals and coral communities of Arabia. Fauna Saudi Arabia 12: 1-170.

Smith, D.B. 1991. The reproduction and recruitment of Porites panamensis Verrill at Uva Island, Pacific Panama. Tesis MSc, University of Miami, Miami, Florida. 65 p.

Steiner, S.C.C.\& J. Cortés. 1996. Spermatozoanultrastructure of scleractinian corals from the eastern Pacific: Pocilloporidae and Agariciidae. Coral Reefs 15: 143-147.

Veron, J.E.N. 1986. Corals of Australia and the Indo-Pacific. Angus \& Robertson, Londres. 644 p.

Veron, J.E.N. 1992. Hermatypic corals of Japan. Aust. Inst. Mar. Sci., Mon. Ser. 9: 1-244.

Veron, J.E.N. 1993. A biogeographic database of hermatypic corals, species of the central Indo-Pacific, genera of the world. Aust. Inst. Mar. Sci., Mon. Ser. 10: 1-433.

Veron, J.E.N. \& G. Hodgson. 1989. Annotated checklist of the hermatypic corals of the Philippines. Pac. Sci. 43: 234287. 
Veron, J.E.N. \& $\dot{R}$. Kelley. 1988. Species stability in reef corals of Papua New Guinea and the Indo Pacific. Ass. Aust. Pal. Mem. 6: 1-69.

Veron, J.E. N. \& M. Pichon. 1976. Scleractinia of Eastern Australia, part 1. Families Thamnasteriidae, Astrocoeniidae, Pocilloporidae. Aust. Inst. Mar. Sci., Mon. Ser. 1: 1-86.

Veron, J.E.N. \& M. Pichon. 1979. Scleractinia of Eastern Australia, part 111. Families Agariciidae, Siderastreidae, Fungiidae, Oculinidae, Merulinidae, Mussidae, Pectiniidae, Caryophlliidae, Dendrophylliodae. Aust. Inst. Mar. Sci., Mon. Ser. 4: 1-471.

Veron, J.E.N. \& M. Pichon. 1982. Scleractinia of Eastern Australia, part IV. Family Poritidae. Aust. Inst. Mar. Sci., Mon. Ser. 5: 1-159.

Veron, J.E.N. \& C.C. Wallace. 1984. Scleractinia of Eastern Australia, part V. Family Acroporidae. Aust. Inst. Mar. Sci., Mon. Ser. 6: 1-485

Veron, J.E. N., M. Pichon \& M. Wijsman-Best. 1977 Scleractinia of Eastern Australia, part ll. Families Faviidae, Trachyliidae. Aust. Inst. Mar. Sci., Mon. Ser. 3: 1-233.
Weil, E. 1992. Genetic and morphological variation in Caribbean and eastern Pacific Porites (Anthozoa, Scleractinia). Preliminary results. Proc. 7th Int. Coral Reef Symp., Guam, 2: 643-656.

Wells, J.W. 1956. Scleractinian corals, part 9. New corals from the Galápagos Islands. Pac. Sci. 36: 211-219.

Wells, J.W. 1982. Notes on Indo-Pacific scleractinian corals. New coral species from the Galapagos Islands. Pac. Sci. 36: $211-219$.

Wells, J.W. 1983. Annoted list of the scleractinian corals of the Galápagos, p. 211-29I. In P.W. Glynn and G.M. Wellington, Corals and Coral Reefs of the Galápagos Islands. University of California, Berkeley.

Yamashiro, H. 1992. Skeletal dissolution by scleractinian corals. Proc. 7th Int. Coral Reef Symp., Guam 2: 11421146.

Yamashiro, H. \& M. Nishihira. 1994. Radial skeletal dissolution to promote vegetative reproduction in a solitary coral Diaseris distorta. Experientia 50: 497-498. 